Draft VERSion MARCh 3, 2021

Typeset using LATEX twocolumn style in AASTeX63

\title{
A coplanar circumbinary protoplanetary disk in the TWA 3 triple M dwarf system
}

\author{
Ian Czekala, $, 1,2,3,4,5, *$ Álvaro Ribas,${ }^{6}$ Nicolás Cuello, ${ }^{7}$ Eugene Chiang, ${ }^{5,8}$ Enrique Macías, ${ }^{9,6}$ \\ Gaspard Duchêne, ${ }^{5,7}$ Sean M. Andrews, ${ }^{10}$ and Catherine C. Espaillat ${ }^{11}$ \\ ${ }^{1}$ Department of Astronomy and Astrophysics, 525 Davey Laboratory, The Pennsylvania State University, University Park, PA 16802, \\ $U S A$ \\ ${ }^{2}$ Center for Exoplanets and Habitable Worlds, 525 Davey Laboratory, The Pennsylvania State University, University Park, PA 16802, \\ $U S A$ \\ ${ }^{3}$ Center for Astrostatistics, 525 Davey Laboratory, The Pennsylvania State University, University Park, PA 16802, USA \\ ${ }^{4}$ Institute for Computational 83 Data Sciences, The Pennsylvania State University, University Park, PA 16802, USA \\ ${ }^{5}$ Department of Astronomy, 501 Campbell Hall, University of California, Berkeley, CA 94720-3411, USA \\ ${ }^{6}$ European Southern Observatory (ESO), Alonso de Córdova 3107, Vitacura, Casilla 19001, Santiago de Chile, Chile \\ ${ }^{7}$ Univ. Grenoble Alpes, CNRS, IPAG (UMR 5274), F-38000 Grenoble, France \\ ${ }^{8}$ Department of Earth and Planetary Science, University of California at Berkeley, Berkeley, CA 94720-4767, USA \\ ${ }^{9}$ Joint ALMA Observatory, Alonso de Córdova 3107, Vitacura, Santiago, Chile \\ ${ }^{10}$ Center for Astrophysics | Harvard 85 Smithsonian, 60 Garden Street, Cambridge, MA 02138, USA \\ ${ }^{11}$ Department of Astronomy, Boston University, 725 Commonwealth Avenue, Boston, MA 02215, USA
}

\section{ABSTRACT}

We present sensitive ALMA observations of TWA 3, a nearby, young ( 10 Myr) hierarchical system composed of three pre-main sequence M3-M4.5 stars. For the first time, we detected ${ }^{12} \mathrm{CO}$ and ${ }^{13} \mathrm{CO} J=2-1$ emission from the circumbinary protoplanetary disk around TWA 3A. We jointly fit the protoplanetary disk velocity field, stellar astrometric positions, and stellar radial velocities to infer the architecture of the system. The Aa and Ab stars $\left(0.29 \pm 0.01 M_{\odot}\right.$ and $0.24 \pm 0.01 M_{\odot}$, respectively) comprising the tight $(P=35$ days $)$ eccentric $(e=0.63 \pm 0.01)$ spectroscopic binary are coplanar with their circumbinary disk (misalignment $<6^{\circ}$ with $68 \%$ confidence), similar to other short-period binary systems. From models of the spectral energy distribution, we found the inner radius of the circumbinary disk $\left(r_{\text {inner }}=0.50-0.75 \mathrm{au}\right)$ to be consistent with theoretical predictions of dynamical truncation $r_{\text {cav }} / a_{\text {inner }} \approx 3$. The outer orbit of the tertiary star $\mathrm{B}\left(0.40 \pm 0.28 M_{\odot}, a \sim 65 \pm 18 \mathrm{au}\right.$, $e=0.3 \pm 0.2)$ is not as well constrained as the inner orbit, however, orbits coplanar with the A system are still preferred (misalignment $<20^{\circ}$ ). To better understand the influence of the $\mathrm{B}$ orbit on the TWA 3A circumbinary disk, we performed SPH simulations of the system and found that the outer edge of the gas disk $\left(r_{\text {outer }}=8.5 \pm 0.2 \mathrm{au}\right)$ is most consistent with truncation from a coplanar, circular or moderately eccentric orbit, supporting the preference from the joint orbital fit.

Keywords: protoplanetary disks - stars: pre-main sequence - orbits - classical T Tauri stars - Trinary stars - M dwarf stars

\section{INTRODUCTION}

The distribution of pre-main sequence multiple system architectures informs our understanding of the mechanisms that govern star and planet formation. Recently, Czekala et al. (2019) found that the degree of alignment between the disk and its host binary (the mutual inclination, $\theta$ ) is a strong function of orbital period. Circumbinary disks around short-period binaries $(P<40$ days $)$

Corresponding author: Ian Czekala

iczekala@berkeley.edu

* NASA Hubble Fellowship Program Sagan Fellow are preferentially coplanar, while disks around longer period binaries exhibit a wide range of mutual inclinations, including polar configurations $\left(\theta \approx 90^{\circ}\right)$. These trends might be manifestations of the same physical mechanisms that produce close binaries. Because it is difficult to directly fragment stars on scales $<5$ au (Larson 1969; Bate et al. 2002; Offner et al. 2016), it is believed that tight binaries are instead produced from wider binaries that have hardened through star-disk interactions (Offner et al. 2010; Bate 2019). What mechanisms mediate this evolution, whether migration affects mutual inclination, and whether initial mutual inclination affects migration efficiency are all open questions. 
Typically, a nearly coplanar disk surrounding a low eccentricity binary will precess around the binary angular momentum vector as dissipative forces damp the angular momentum vectors of the disk and the binary into alignment (Foucart \& Lai 2013). If the binary is sufficiently eccentric, however, then the disk can precess around the eccentricity vector ${ }^{1}$ (Aly et al. 2015; Martin \& Lubow 2017; Zanazzi \& Lai 2018; Cuello \& Giuppone 2019) and access polar mutual inclinations. Indeed, highly misaligned disks are preferentially found around highly eccentric $(e>0.7)$ binaries (Kennedy et al. 2019; Czekala et al. 2019). There are, however, several disks around eccentric short period binaries that are coplanar, such as AK Sco, DQ Tau, and UZ Tau E (Czekala et al. 2015, 2016, 2019). Our understanding of how these period and eccentricity trends interrelate is limited by the sample size of circumbinary disk systems with well-measured architectures.

The pre-main sequence system TWA 3 represents an opportunity to expand this sample to aid in the interpretation of binary formation and evolution mechanisms. TWA 3 consists of three young $(10 \pm 3 \mathrm{Myr}$; Bell et al. 2015), pre-main sequence M3-M4.5 stars in a hierarchical configuration; their spectral types correspond to $\sim 0.3 M_{\odot}$ (Herczeg \& Hillenbrand 2014; Tofflemire et al. 2019). The inner Aa-Ab binary has an orbital period of $P=34.8785 \pm 0.0009$ days, an eccentricity of $e=0.628 \pm 0.006$, and spectral types of M4 and M4.5, respectively (Kellogg et al. 2017). The Gaia DR2 parallax is $\varpi=27.31 \pm 0.12$ mas (including a 0.02 mas systematic term, Lindegren et al. 2018) corresponding to a distance of $36.62 \pm 0.16$ pc (Gaia Collaboration et al. 2018; Bailer-Jones et al. 2018). Tofflemire et al. (2019) noted that the $\mathrm{A}$ and $\mathrm{B}$ components suffer from significant excess astrometric noise-possibly due to photometric variability - but the parallax distances for each source are consistent with each other and the A-B orbit arcs in Kellogg et al. (2017). Time-series photometry and emission line spectroscopy revealed that accretion from the circumbinary disk to the inner binary is phased with periastron, and that material is preferentially accreted onto the primary star, TWA 3Aa (Tofflemire et al. 2017, 2019). The gradual movement of the outer triple companion (projected separation 1".55 or $57 \mathrm{au}$; Tokovinin et al. 2015) over a $\sim 20 \mathrm{yr}$ baseline suggests an orbital period of $\sim 200-800 \mathrm{yr}$ (Kellogg et al. 2017).

Andrews et al. (2010) used the Submillimeter Array (SMA) to localize the submillimeter emission in the TWA 3 system to the A binary, measuring a flux density of $75 \mathrm{mJy}$ at $340 \mathrm{GHz}$. As demonstrated by fits to the deprojected and azimuthally averaged baselines, the circumbinary disk itself was only marginally resolved ( $1^{\prime \prime}$. 11 $\times 00^{\prime \prime} 74$ beam), but found to have a radius of $\sim 20 \mathrm{au}$. Andrews et al. (2010) did not detect ${ }^{12} \mathrm{CO} J=3-2$

${ }^{1}$ The eccentricity vector is drawn from binary apoapse to periapse. emission to an upper limit of $0.6 \mathrm{Jy}_{\text {beam }}{ }^{-1}$ integrated over a $0.7 \mathrm{~km} \mathrm{~s}^{-1}$ channel. Based upon the fit of an elliptical Gaussian to the visibilities, Andrews et al. (2010) derived a disk inclination of $i_{\text {disk }}=36^{\circ} \pm 10^{\circ}$ (relative to the sky plane) and disk orientation of $\Omega_{\text {disk }}=169^{\circ} \pm 15^{\circ}$ (the position angle of the ascending node measured east of north). ${ }^{2}$

More recent orbital solutions suggested that the inner binary orbit, circumbinary disk, and outer tertiary orbit may be misaligned. Kellogg et al. (2017) combined an astrometric observation of the inner binary (Anthonioz et al. 2015) with their double-lined radial velocity solution to constrain the position angle of the ascending node $\Omega_{\text {inner }} \in\left[93^{\circ}, 123^{\circ}\right]$ and inclination $i_{\text {inner }} \in\left[32^{\circ}, 63^{\circ}\right]$ or $i_{\text {inner }} \in\left[118^{\circ}, 149^{\circ}\right]$. These orbital parameters, together with the disk parameters reported in Andrews et al. (2010), suggested that the planes of the spectroscopic binary and the circumbinary disk were misaligned by at least $\theta \sim 30^{\circ}$ (Kellogg et al. 2017).

We acquired ALMA observations of the TWA 3A circumbinary disk to better understand its size and orientation relative the stellar orbits. In $\S 2$ we describe the ALMA observations and data reduction. In $\S 3$ we dynamically model the gas rotation curve of the TWA 3A circumbinary disk, fit the spectral energy distribution (SED) of TWA 3A, and perform a joint stellar orbital fit to the radial velocities and astrometric measurements of the $\mathrm{Aa}, \mathrm{Ab}$, and $\mathrm{B}$ stars. In $\S 4$ we describe our smoothed-particle hydrodynamics (SPH) simulations of the TWA 3 system and discuss how they support our interpretation of the circumbinary disk as nearly coplanar and dynamically truncated both internally and externally by binary companions. We also briefly review similar analog protoplanetary and exoplanetary systems in our discussion of mutual inclination and disk truncation. We conclude in $\S 5$.

\section{DATA}

We obtained deep Atacama Large Millimeter Array (ALMA) observations of the TWA 3 system in 2018. We used a correlator setup that assigned two $2 \mathrm{GHz}$ wide spectral windows to the dust continuum (centered on $220 \mathrm{GHz}$ and $232 \mathrm{GHz}$ ) and two spectral windows at $122 \mathrm{kHz}\left(0.16 \mathrm{~km} \mathrm{~s}^{-1}\right)$ resolution to target the ${ }^{12} \mathrm{CO}$ and ${ }^{13} \mathrm{CO} J=2-1$ transitions. Two sets of observations (project code 2018.1.01545.S) were executed on Oct 16th and 27th, 2018 (JD 2458408.1404 and 2458419.0483, respectively) using 44 and 43 antennas of the main array, respectively. The array was similarly configured for each observation, with baselines ranging from $15 \mathrm{~m}$ to $2.4 \mathrm{~km}$. Both observing sequences used the J1107-4449 quasar as an amplitude and bandpass calibrator and used the J1126-3828 quasar as a phase calibrator. Each execution

\footnotetext{
2 The lack of a gas detection meant degenerate "flipped" solutions were also valid: $i_{\text {disk }}=144^{\circ} \pm 10^{\circ}$ and $\Omega_{\text {disk }}=349^{\circ} \pm 15^{\circ}$.
} 
Table 1. Image properties

\begin{tabular}{lcc}
\hline \hline & beam dimensions, P.A. & RMS $\left[\mathrm{mJy} \mathrm{beam}^{-1}\right]$ \\
\hline $226 \mathrm{GHz}$ cont. & $0^{\prime \prime} .24 \times 00^{\prime \prime} 19,-64^{\circ}$ & 0.013 \\
${ }^{12} \mathrm{CO} J=2-1$ & $0^{\prime \prime} .24 \times 00^{\prime \prime} 19,-64^{\circ}$ & 1.1 \\
${ }^{13} \mathrm{CO} J=2-1$ & $0^{\prime \prime} .25 \times 00^{\prime \prime} 20,-63^{\circ}$ & 1.3 \\
\hline
\end{tabular}

Note-The RMS noise levels for the spectral line cubes correspond to the values per $0.8 \mathrm{~km} \mathrm{~s}^{-1}$ channel. All images were synthesized with robust $=0.5$.

spent 47.4 minutes on-source, for a total on-source time of $1 \mathrm{hr} 34.8 \mathrm{~m}$. The mean precipitable water vapor for each observation was $1.9 \mathrm{~mm}$ and $0.4 \mathrm{~mm}$, respectively.

We began our data reduction with the pipeline-calibrated measurement set provided by ALMA/NAASC staff. We used the CASA 5.4 (McMullin et al. 2007) facility software and followed common calibration and imaging procedures (e.g., the DSHARP reduction scripts, Andrews et al. 2018) ${ }^{3}$, the pertinent details of which we now describe. To assess the quality of each execution block we first reduced each observation individually. We excised the channels with line-emission to create a continuum-only measurement set with a total bandwidth of $4.6 \mathrm{GHz}$. We performed an initial round of continuum imaging using the CASA tclean task with robust $=0.5$, an image size of $512 \times 512$ pixels, $0^{\prime \prime} 015$ pixel size, deconvolver="multiscale", scales $=[0,15,30,45,75]$ pixels, a threshold of $0.6 \mathrm{mJy}$, and an elliptical mask with position angle $110^{\circ}$, semi-major axis of $0{ }^{\prime \prime} 45$ and semi-minor axis of $0 " 36$.

We fit the continuum emission with an elliptical Gaussian using the imfit and uvmodelfit tasks. We found excellent astrometric agreement between executions, with the emission centroid located at ICRS 11:10:27.731 -37.31.51.84, coincident with the Gaia position of TWA 3A to within $0{ }^{\prime \prime} 05$. We found adequate agreement between the total continuum fluxes $(31.9 \mathrm{mJy}$ and $36.1 \mathrm{mJy}$, respectively), only slightly more different than the expected $10 \%$ amplitude calibration uncertainty. ${ }^{4}$

We proceeded to self-calibrate the combined measurement set through a series of applications of tclean to the continuum visibilities using threshold depths $\{0.6$, 0.15 , and 0.15$\} \mathrm{mJy}^{-1}$ beam ${ }^{-1}$ interleaved with applications of the gaincal and applycal CASA tasks using

\footnotetext{
3 https://bulk.cv.nrao.edu/almadata/lp/DSHARP/

${ }^{4}$ See the ALMA Technical Handbook (Remijan et al. 2019) and ALMA Memo 594 https://science.nrao.edu/facilities/ alma/aboutALMA/Technology/ALMA_Memo_Series/alma594/ memo594.pdf
}

spectral-window dependent solves with intervals $\{60 \mathrm{~s}$, $30 \mathrm{~s}$, and $18 \mathrm{~s}\}$. We then cleaned to a final depth of $0.02 \mathrm{mJy}_{\text {beam }}{ }^{-1}$ and performed one round of phase and amplitude gain solutions over the scan length (8 minutes, solint='inf'). We monitored the peak flux, total flux, and RMS of the images throughout the process (Brogan et al. 2018) and found the peak continuum S/N improved to 2130 from an initial value of 260 .

We then used the applycal task to apply the selfcalibration solutions to channels containing the spectral line observations ${ }^{5}$ (without propagating flags for failed solutions, applymode=calonly). We estimated the continuum from nearby line-free channels and subtracted it from the spectral line observations using the uvcontsub CASA task. The line channels were imaged using the tclean task with the auto-multithresh masking algorithm with cell="0.015arcsec", gain=0.1, deconvolver="multiscale", scales $=[0,10,30$, $100,200,300]$ pixels, robust $=0.5$, and deconvolved to a depth of threshold="0.1mJy". The beam dimensions (FWHM) and image-plane RMS are described in Table 1.

We summed the pixels within the continuum CLEAN mask to measure a total continuum flux of $36.7 \mathrm{mJy}$. The dust continuum emission is compact, with nearly all of the flux contained within the central beam. We fit elliptical Gaussians to the continuum emission with the uvmodelfit and imfit tasks and derived FWHM dimensions of $\left(0.12 \times 0.0^{\prime \prime} 12\right)$ and $\left(0.16 \times 00^{\prime \prime} 10\right)$, respectively. Since these dimensions are on the order of the beam size, to better quantify whether we resolved the dust continuum we also deprojected and azimuthally averaged the continuum visibilities using the uvplot package (Tazzari 2017), shown in Figure 2. We used deprojection values of $i_{\text {disk }}=49^{\circ}$ and $\Omega_{\text {disk }}=116.5^{\circ}$, which were derived from our modeling effort of the ${ }^{12} \mathrm{CO}$ $J=2-1$ line emission, since the more extended emission provided better constraints for these parameters than the dust continuum emission (see §3.1). The declining visibility profile demonstrates that the outer extent of the disk is resolved: if the deprojected continuum emission profile is represented by a Gaussian, then a Fourierdomain $\mathrm{FWHM} \approx 1200 \mathrm{k} \lambda$ implies that the image plane Gaussian has FWHM $\approx 00^{\prime \prime} 15$, or 5.5 au.

In preliminary line imaging, we identified ${ }^{12} \mathrm{CO}$ emission from $[-9,10] \mathrm{km} \mathrm{s}^{-1}$ (LSRK). To save computational complexity, we used the task mstransform to average the channels to $0.8 \mathrm{~km} \mathrm{~s}^{-1}$ width. The ${ }^{12} \mathrm{CO}$ emission was strongly detected across this velocity range (peak channel $\mathrm{S} / \mathrm{N}=30$, see Figure 3, top panel); the ${ }^{13} \mathrm{CO}$

\footnotetext{
${ }^{5}$ Though part of a standard self-calibration workflow, we noticed that the $\mathrm{S} / \mathrm{N}$ of the line channels did not measurably improve after applying the self-calibration solutions. We believe that this is because the fine resolution channels are thermal-noise dominated and not limited by residual phase errors.
} 


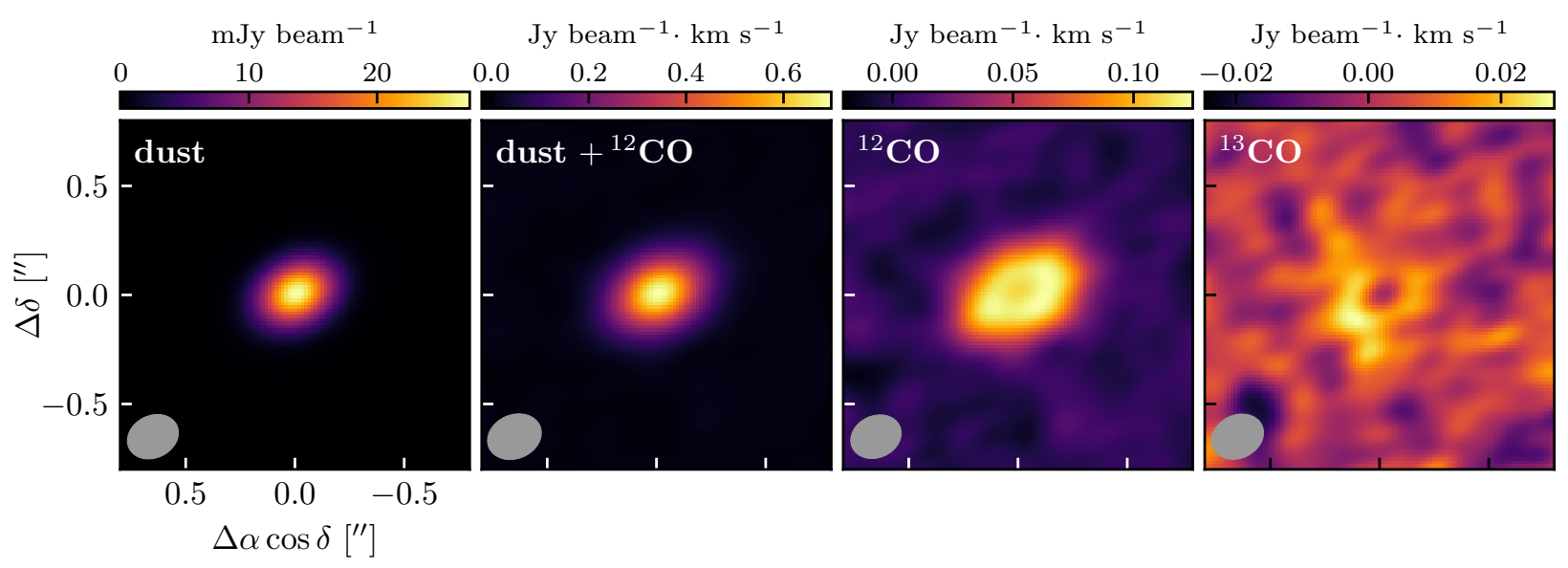

Figure 1. from left to right: the $226 \mathrm{GHz}$ dust continuum; the channels centered on the ${ }^{12} \mathrm{CO}$ emission (including the dust continuum) integrated over the full range of the ${ }^{12} \mathrm{CO}$ emission; the continuum-subtracted ${ }^{12} \mathrm{CO}$ emission; and the continuum-subtracted ${ }^{13} \mathrm{CO}$ emission. The FWHM beam is shown in the lower left of each panel. Full channel maps for ${ }^{12} \mathrm{CO}$ and ${ }^{13} \mathrm{CO}$ are in $\S 3.1$ and the Appendix, respectively.

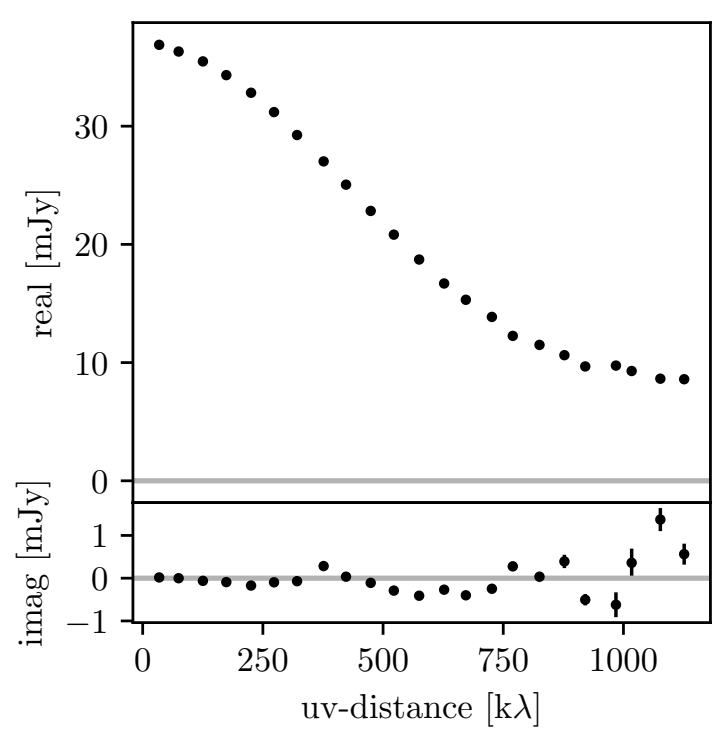

Figure 2. The continuum visibilities deprojected from the disk inclination. That the flux drops with increasing uv distance indicates that the continuum emission is spatially resolved. The Gaussian profile with $\mathrm{FWHM} \approx 1200 \mathrm{k} \lambda$ implies an image plane morphology with FWHM $\approx 0$ '. 15 , or $5.5 \mathrm{au}$.

emission was detected at lower but still significant levels (peak channel $\mathrm{S} / \mathrm{N}=6$, see Appendix $\mathrm{A}$ ). We used the immoments CASA task to sum all flux in each channel across the velocity dimension, producing the moment maps in the third and fourth panels of Figure 1. We used the imstat CASA task to sum all flux within the CLEAN mask across the velocity and and spatial dimensions, yielding integrated fluxes of $526 \mathrm{mJy} \mathrm{km} \mathrm{s}^{-1}$ and $86 \mathrm{mJy} \mathrm{km} \mathrm{s}^{-1}$ for ${ }^{12} \mathrm{CO}$ and ${ }^{13} \mathrm{CO}$, respectively.
We noticed a central cavity in the moment maps of the continuum-subtracted line emission, corresponding to the location of peak dust continuum. The depression is most apparent in the ${ }^{12} \mathrm{CO}$ emission but is also visible in the ${ }^{13} \mathrm{CO}$ emission (Figure 1, third and fourth panels). To investigate whether this may be a continuum subtraction artefact, we produced channel maps and a moment map for the non-continuum subtracted ${ }^{12} \mathrm{CO}$ spectral channels (Figure 1, second panel). This moment map does not exhibit a central cavity, suggesting that the feature seen in ${ }^{12} \mathrm{CO}$ and ${ }^{13} \mathrm{CO}$ is indeed a continuum subtraction artefact. In protoplanetary disks, such an artefact can arise when gas emission on the nearside of the disk is optically thick and absorbs continuum emission originating from the disk midplane. When the continuum emission (estimated from channels offset in velocity from the line emission) is subtracted, most if not all of the line flux is also (erroneously) subtracted (for a full description of the effect, see Weaver et al. 2018). For this artefact to be significant, the continuum emission needs to have a brightness temperature comparable to the line emission, suggesting that the continuum emission is also optically thick, or nearly so.

No noticeable continuum or gas emission is present in the system beyond the disk surrounding TWA 3A. We used an aperture approximately three times the area of the beam to extract continuum photometry at the location of B (see §3.3; Mason et al. 2018), and did not detect anything $(37 \pm 27 \mu \mathrm{Jy})$.

\section{ANALYSIS}

\subsection{Dynamical gas analysis}

Following the approach in Czekala et al. (2015), we constructed a forward model of the continuumsubtracted ${ }^{12} \mathrm{CO} J=2-1$ visibilities to derive constraints on the disk architecture and velocity field. Briefly, a 3D model of the disk density, temperature, 


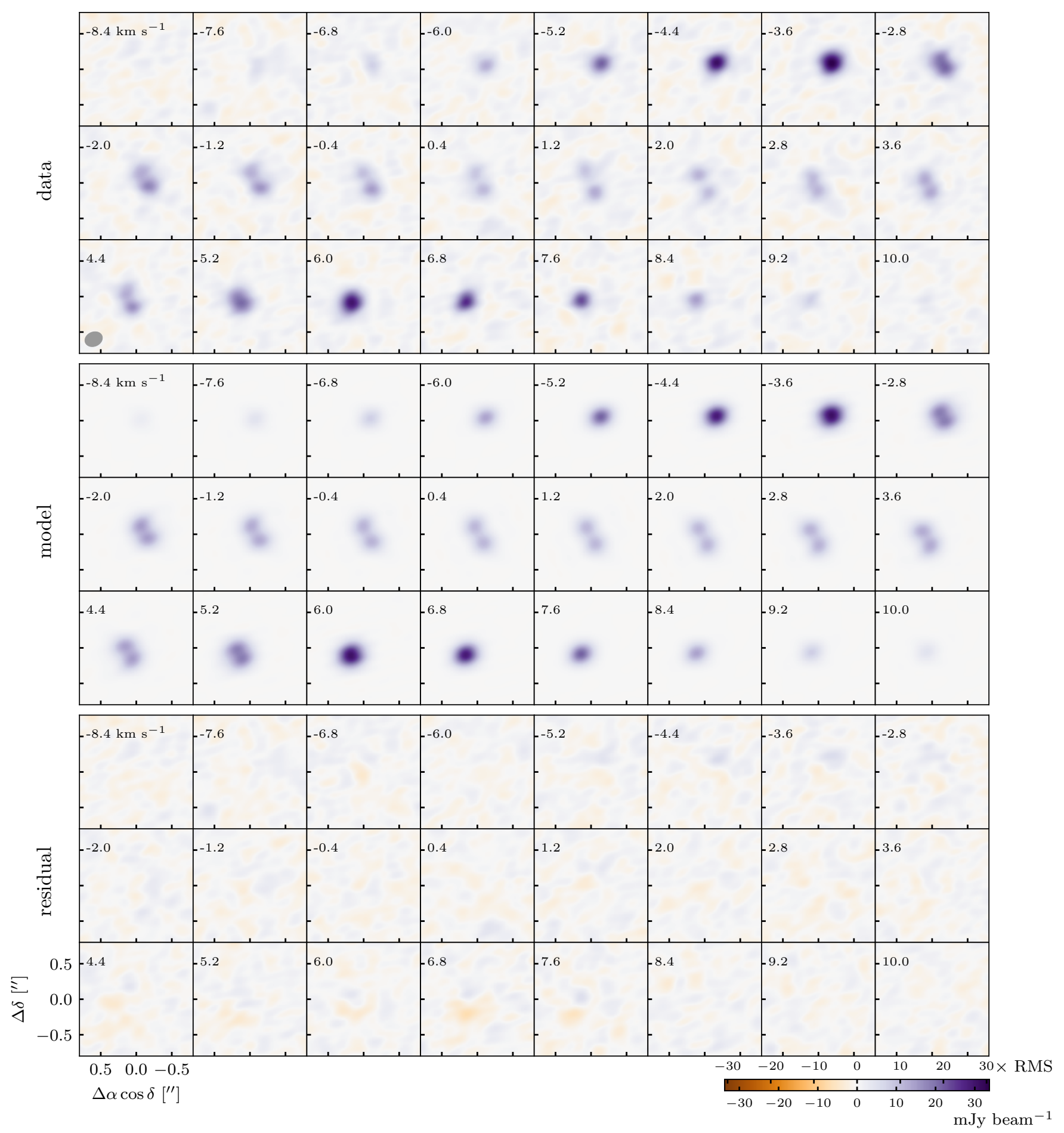

Figure 3. Continuum-subtracted ${ }^{12} \mathrm{CO} J=2-1$ data, model, and residual channel maps. Model and residual visibilities were imaged the same way as the data. Velocity scale is labeled in the LSRK frame. 
and gas model simultaneously fit to the dust continuum and ${ }^{12} \mathrm{CO} J=2-1$ emission could in principle recover a more accurate gas surface density profile. However, the behavior of the surface density profile at small radii is effectively a nuisance component to our dynamical analysis and does not justify a more sophisticated model, especially since its significantly expanded computational requirements would curtail our ability to thoroughly explore the posterior distributions of key parameters $\left(M_{\mathrm{A}}\right.$, $i_{\text {disk }}$, and $\left.\Omega_{\text {disk }}\right)$ via MCMC.

We constrained the disk position angle to be $\Omega_{\text {disk }}=$ $116.5^{\circ} \pm 0.4^{\circ}$, which is significantly different from the value found by Andrews et al. (2010) $\left(\Omega_{\text {disk }}=144^{\circ} \pm\right.$ $\left.10^{\circ}\right)$. We constrained the disk inclination to be either $i_{\text {disk }}=48.8^{\circ} \pm 0.7^{\circ}$ or $i_{\text {disk }}=131.2^{\circ} \pm 0.7^{\circ}$, which is also in disagreement with the values found by Andrews et al. $(2010)\left(i_{\text {disk }}=36^{\circ} \pm 10^{\circ}\right.$ or $\left.i_{\text {disk }}=144^{\circ} \pm 10^{\circ}\right)$. Andrews et al. (2010) derived the disk inclination and position angle by fitting an elliptical Gaussian to marginally resolved sub-mm continuum observations, so it is only mildly surprising that their simplistic model deviates from the new values we derived using a more realistic dynamical gas model fit to higher quality observations. We constrained the central stellar mass to be $M_{\mathrm{A}}=M_{\mathrm{Aa}}+$ $M_{\mathrm{Ab}}=0.534 \pm 0.010 M_{\odot}$ and the systemic velocity of the TWA 3A circumbinary disk to be $1.22 \pm 0.02 \mathrm{~km} \mathrm{~s}^{-1}$ in the LSRK frame $\left(10.48 \pm 0.02 \mathrm{~km} \mathrm{~s}^{-1}\right.$ in the BARY frame). ${ }^{7}$ This is fully consistent with the radial velocity of the TWA 3A barycenter $v_{\text {LSRK }}=0.91 \pm 0.40 \mathrm{~km} \mathrm{~s}^{-1}$ $\left(\gamma_{\mathrm{A}}=10.17 \pm 0.40 \mathrm{~km} \mathrm{~s}^{-1}\right.$ on the CfA system, Kellogg et al. 2017).

\subsection{SED modeling and (sub)mm spectral index}

In light of our new $226 \mathrm{GHz}$ ALMA flux density measurement, we updated the spectral energy distribution (SED) of the TWA 3A system to learn about the properties of the circumbinary disk and its interior cavity. We sourced photometric fluxes from the SED compilation in Kellogg et al. (2017). We also incorporated the mid-IR spectrum from the IRS spectrograph onboard the Spitzer Space Telescope (Andrews et al. 2010) with the contribution from the B component subtracted. The SED (shown in Figure 4) continuously decreases redward of $\sim 20 \mu \mathrm{m}$, suggesting that the circumbinary disk is truncated at larger radii, highly settled, or both. The observed spectral slope between the SMA $880 \mu \mathrm{m}$ and the $1.3 \mathrm{~mm}$ ALMA data is:

$$
\alpha=\frac{\mathrm{d} \log F_{\nu}}{\mathrm{d} \log \nu}=1.7 \pm 0.3,
$$

where the uncertainty is calculated by adopting a $10 \%$ calibration uncertainty for both points. That the spectral index is $\alpha \lesssim 2$ suggests the dust continuum emission is optically thick at these wavelengths, consistent with

\footnotetext{
${ }^{7}$ In the direction of TWA $3, v_{\mathrm{LSRK}}=v_{\mathrm{BARY}}-9.26 \mathrm{~km} \mathrm{~s}^{-1}$.
}

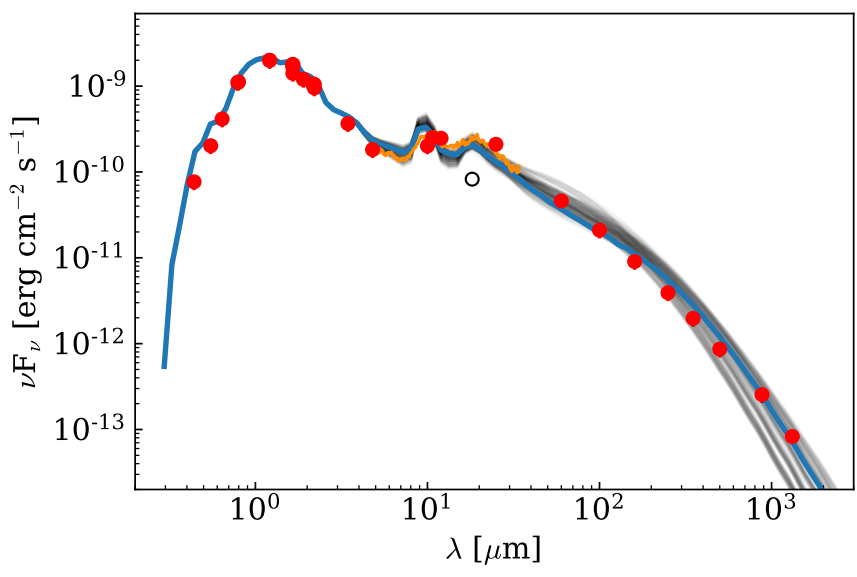

Figure 4. The SED of TWA 3A: Photometric observations are shown in red, and the Spitzer/IRS spectrum in orange. The Qband observation (black empty circle) from Jayawardhana et al. (1999) was not used during the fitting. The best-fit model is shown in blue along with the 100 highest likelihood SED models (grey).

the continuum subtraction artifact described in $\S 2$. If the maximum grain size in the disk is between $500 \mu \mathrm{m}$ and $1 \mathrm{~cm}$, self-scattering from high albedo dust grains can reduce the (sub)mm-wave emission from an optically thick region and produce $\alpha<2$ (Zhu et al. 2019).

Motivated by the near-infrared dip $(\lambda \sim 10 \mu \mathrm{m})$ in the SED, we followed Andrews et al. (2010) and constructed a simple disk model with two zones: an inner cavity with a constant surface density from $r_{\text {in }}$ to $r_{\text {cav }}$, and a disk with surface density profile $\Sigma(r) \propto 1 / r$ from $r_{\text {cav }}$ to $r_{\text {out }}$. We fixed $r_{\text {in }}$ to $0.2 \mathrm{au}$, following Kellogg et al. (2017)'s constraint on the inner binary semi-major axis of $0.19 \mathrm{au}$. We fixed $r_{\text {out }}=8.5 \mathrm{au}$ and $i_{\text {disk }}=49^{\circ}$ following our analysis of the ${ }^{12} \mathrm{CO} J=2-1$ line emission. Our model had five free parameters: total disk dust mass $M_{\text {dust }}$, cavity radius $r_{\text {cav }}$, cavity depletion factor $\delta$, flaring parameter $\beta$, and scale height at 10 au $H_{10}$. The surface density within the cavity $\left(r_{\text {in }}<r<r_{\text {cav }}\right)$ was set to a constant surface density $\Sigma_{\text {cav }}=\delta \Sigma_{\text {disk }}\left(r_{\text {cav }}\right)$. The scale height as a function of radius is defined as

$$
H(r)=H_{10}\left(\frac{r}{10 \mathrm{au}}\right)^{\beta}
$$

We followed the approach in Andrews et al. (2010) and combined the $\mathrm{Aa}$ and $\mathrm{Ab}$ components into a single stellar photosphere with an effective temperature of $3350 \mathrm{~K}$ and radius of $1 \mathrm{R}_{\odot}$ (updated to the new Gaia distance), equivalent to $0.11 \mathrm{~L}_{\odot}$. We adopted the dust composition values used in Pinte et al. (2016): the grain size distribution followed $\mathrm{d} n / \mathrm{d} a \propto a^{-3.5}$, where $a$ is the grain size. The distribution ranged from $a_{\min }=0.01 \mu \mathrm{m}$ to $a_{\max }=1 \mathrm{~cm}$. We computed model SEDs using the MCFOST radiative transfer code (Pinte et al. 2006) assuming no interstellar extinction (McJunkin et al. 2014). Our grid of models spanned parameter ranges $M_{\text {dust }}$ : 
$[0.5,1,2,4,8,16,32,64,128] \times 10^{-6} M_{\odot} ; r_{\text {cav }}:[0.2$, $0.5,0.75,1,1.5,2]$ au; $\delta: 1 /[1,10,100,1000] ; \beta:[1.025$, $1.05,1.075]$; and $H_{10}:[0.2,0.3,0.4,0.5,0.6]$ au.

For each model, we calculated a $\chi^{2}$ figure of merit using the observed photometry and IRS spectrum. Since we were only interested in disk properties, we only used photometric points $\lambda>1 \mu \mathrm{m}$ in the following SED fit. We also excluded the Q-band $(18.2 \mu \mathrm{m})$ observation from Jayawardhana et al. (1999) from the fit since it is an outlier compared to the other photometric points. A set of consistently calibrated photometric and spectroscopic covariance matrices does not exist for the TWA 3A spectroscopic dataset, and so we were unable to use per-datapoint flux uncertainties in our construction of a $\chi^{2}$ fit metric. Instead, we explored the consistency of the model grid with the SED data by the following procedure.

First, because adjacent pixels in spectroscopic fluxes are frequently correlated due to residual calibration errors, we subsampled the spectrum and only fit every third point. Then, we explored the consistency of the model grid with the SED data by assigning relative uncertainties of $5 \%, 10 \%$, and $20 \%$ for each photometric and spectroscopic datapoint and calculated the $\chi^{2}$ metric. We found that $r_{\text {cav }}=0.20$ au was excluded at high significance ( $>99 \%$ probability) for all choices of uncertainty reweighting factors. The $20 \%$ reweighting for both photometric and spectroscopic datasets yielded the lowest reduced $\chi_{\nu}^{2}$ of 1.16. Based on the models with high figures of merit (see Figure 4) we conclude that the disk around TWA $3 \mathrm{~A}$ has a dust mass of $1-8 \times 10^{-6} M_{\odot}$ and a disk cavity $r_{\text {cav }}$ of $\approx 0.5-0.75 \mathrm{au}$. The other model parameters were not well constrained.

\subsection{Stellar orbits}

From the literature, we collected a diverse orbital dataset including radial velocity and astrometric measurements of all three stars in the TWA 3 hierarchical triple (Reipurth \& Zinnecker 1993; Webb et al. 1999; Weintraub et al. 2000; Brandeker et al. 2003; Correia et al. 2006; Janson et al. 2014; Tokovinin et al. 2015; Anthonioz et al. 2015; Kellogg et al. 2017; Knapp \& Nanson 2018; Mason et al. 2018). ${ }^{8}$ Our goal was to extend the comprehensive analysis of Kellogg et al. (2017) by incorporating the new disk-based dynamical constraint on $M_{A}$ (see §3.1) into a joint hierarchical triple fit with the extant radial velocity and astrometric datasets and Gaia parallax.

We modelled these diverse datasets using the exoplanet software package (Foreman-Mackey et al. 2020). Briefly, exoplanet is designed to unify routines needed for orbital parameter inference within the PyMC3 (Salvatier et al. 2016) framework. Posterior gradients

\footnotetext{
${ }^{8}$ Following Kellogg et al. (2017), we assigned a date of 1992.0216 to the observation by Reipurth \& Zinnecker (1993).
}

are provided through the Theano framework (Theano Development Team 2016), enabling the usage of powerful MCMC samplers like Hamiltonian Monte Carlo (HMC; Hoffman \& Gelman 2011) to efficiently explore high dimensional spaces. To fit the TWA 3 datasets, we extended exoplanet to include functionality for astrometric orbits; these routines have been available in the main exoplanet package as of v0.2.0. We constructed the hierarchical model by nesting a Keplerian orbit for $\mathrm{Aa}-\mathrm{Ab}$ ("inner") inside of a wider orbit for A-B ("outer"). In the following analysis, we adopted orbital conventions where the argument of periastron $\omega$ is reported as the value of the "primary" star and $\Omega$ describes the position angle of the ascending node, which is the node where the secondary is receding from the observer. For the inner orbit, $\omega_{\mathrm{Aa}}$ refers to the argument of periastron of TWA $3 \mathrm{Aa}$ and $\Omega_{\text {inner }}$ refers to the position angle of the TWA $3 \mathrm{Aa}-\mathrm{Ab}$ ascending node. For the outer orbit, $\omega_{\mathrm{A}}$ refers to the argument of periastron of TWA 3A (under the assumption that the $\mathrm{Aa}$ and $\mathrm{Ab}$ stars can be treated as a single star, A) and $\Omega_{\text {outer }}$ refers to the position angle of the TWA $3 \mathrm{~A}-\mathrm{B}$ ascending node.

Following standard radial velocity analysis, we included "jitter" and offset terms for each of the instruments. In keeping with Kellogg et al. (2017), we derived orbital parameters using the CfA radial velocity reference scale. Because there may be a small but unknown systematic radial velocity offset between the CfA and ALMA velocity scales, we did not use the systemic velocity of the TWA 3A circumbinary disk (at the epoch of the ALMA measurement) in the joint model. We applied uniform priors on the following quantities: $\log P_{\text {inner }}, \log P_{\text {outer }}, \cos i_{\text {inner }}, \cos i_{\text {outer }}$, and $\log$ jitter terms. We applied broad Gaussian priors on the sampled stellar masses of $M_{\mathrm{Ab}}$ of $0.29 \pm 0.50 M_{\odot}$ and $M_{\mathrm{B}}$ of $0.3 \pm 0.5 M_{\odot}$, loosely corresponding to the spectral types, and truncated to positive values only. We also applied broad Gaussian priors of $0.0 \pm 5.0 \mathrm{~km} \mathrm{~s}^{-1}$ on the instrument offset terms.

Table 3 lists a full description of the inferred orbital parameters, where posterior means and standard deviations are provided for parameters whose posteriors are approximately Gaussian. Table 3 covers two scenarios: 1) the "primary" solution where $i_{\text {inner }}>90^{\circ}$ and 2) the "alternate" solution where $i_{\text {inner }}<90^{\circ}$. The alternate solution yields retrograde orbits between the inner and outer orbits. We show the phase-folded spectroscopic binary orbit (identical for both scenarios) in Figure 5, which is in good agreement with that found by Kel$\operatorname{logg}$ et al. (2017). The joint hierarchical fit delivered more precise posteriors for many outer orbital parameters, though some of the degeneracies noted in Kellogg et al. (2017) still remain.

Three outer orbit parameters have bimodal posterior distributions: $\omega_{\mathrm{A}}, \Omega_{\text {outer }}$, and $T_{0, \text { outer }}$. The posteriors for these outer orbit parameters are identical between the primary and alternate solutions, so the single cor- 
Table 3. Orbital parameters

\begin{tabular}{|c|c|c|}
\hline Parameter & primary solution & alternate solution \\
\hline \multicolumn{3}{|l|}{ Sampled } \\
\hline$P_{\text {inner }}[$ days $]$ & $34.879 \pm 0.001$ & $34.879 \pm 0.001$ \\
\hline$a_{\text {inner }}[\mathrm{mas}]$ & $4.63 \pm 0.04$ & $4.63 \pm 0.04$ \\
\hline$M_{\mathrm{Ab}}\left[M_{\odot}\right]$ & $0.24 \pm 0.01$ & $0.24 \pm 0.01$ \\
\hline$e_{\text {inner }}$ & $0.63 \pm 0.01$ & $0.63 \pm 0.01$ \\
\hline$i_{\text {inner }}\left[^{\circ}\right]$ & $131.5 \pm 0.8$ & $48.5 \pm 0.8$ \\
\hline$\left.\omega_{\mathrm{Aa}}^{a}{ }^{\circ}{ }^{\circ}\right]$ & $81 \pm 1$ & $81 \pm 1$ \\
\hline$\left.\Omega_{\text {inner }} b{ }^{\circ}\right]$ & $104 \pm 9$ & $112 \pm 9$ \\
\hline$T_{0, \text { inner }}[\mathrm{JD}-2,450,000]$ & $2704.57 \pm 0.07$ & $2704.57 \pm 0.07$ \\
\hline$P_{\text {outer }}[\mathrm{yrs}]$ & $548 \pm 244$ & $555 \pm 249$ \\
\hline$M_{\mathrm{B}}\left[M_{\odot}\right]$ & $0.41 \pm 0.28$ & $0.40 \pm 0.28$ \\
\hline$e_{\text {outer }}$ & $0.3 \pm 0.2$ & $0.3 \pm 0.2$ \\
\hline$i_{\text {outer }}\left[^{\circ}\right]$ & $139 \pm 13$ & $139 \pm 13$ \\
\hline$\omega_{\mathrm{A}}^{a}\left[^{\circ}\right]$ & $\ldots \quad c$ & $\ldots c$ \\
\hline$\left.\Omega_{\text {outer }} b{ }^{\circ}\right]$ & $\ldots \quad c$ & $\ldots c$ \\
\hline$T_{0, \text { outer }}[\mathrm{JD}-2,450,000]$ & $\ldots \quad c$ & $\ldots c$ \\
\hline$\varpi\left[^{\prime \prime}\right]$ & $27.31 \pm 0.12$ & $27.31 \pm 0.12$ \\
\hline$\sigma_{\rho}\left[{ }^{\prime \prime}\right]$ & $0.009 \pm 0.004$ & $0.009 \pm 0.004$ \\
\hline$\sigma_{\theta}\left[^{\circ}\right]$ & $0.024 \pm 0.008$ & $0.024 \pm 0.008$ \\
\hline$\sigma_{\mathrm{CfA}}\left[\mathrm{km} \mathrm{s}^{-1}\right]$ & $3.8 \pm 0.3$ & $3.8 \pm 0.3$ \\
\hline$\sigma_{\text {Keck }}\left[\mathrm{km} \mathrm{s}^{-1}\right]$ & $0.8 \pm 0.1$ & $0.8 \pm 0.1$ \\
\hline$\sigma_{\text {FEROS }}\left[\mathrm{km} \mathrm{s}^{-1}\right]$ & $3.4 \pm 0.6$ & $3.4 \pm 0.6$ \\
\hline$\sigma_{\text {du Pont }}\left[\mathrm{km} \mathrm{s}^{-1}\right]$ & $2.1 \pm 0.4$ & $2.1 \pm 0.4$ \\
\hline$(\mathrm{Keck}-\mathrm{CfA})\left[\mathrm{km} \mathrm{s}^{-1}\right]$ & $-1.3 \pm 0.5$ & $-1.3 \pm 0.5$ \\
\hline$(\mathrm{FEROS}-\mathrm{CfA})\left[\mathrm{km} \mathrm{s}^{-1}\right]$ & $1.3 \pm 1.0$ & $1.3 \pm 1.0$ \\
\hline$\left(\mathrm{du}\right.$ Pont - CfA) $\left[\mathrm{km} \mathrm{s}^{-1}\right]$ & $-0.2 \pm 0.7$ & $-0.2 \pm 0.7$ \\
\hline
\end{tabular}

Derived

\begin{tabular}{lcc}
\hline$M_{\mathrm{Aa}}\left[M_{\odot}\right]$ & $0.29 \pm 0.01$ & $0.29 \pm 0.01$ \\
$M_{\mathrm{A}}\left[M_{\odot}\right]$ & $0.53 \pm 0.01$ & $0.53 \pm 0.01$ \\
$a_{\text {inner }}[\mathrm{au}]$ & $0.170 \pm 0.001$ & $0.170 \pm 0.001$ \\
$a_{\text {outer }}[\mathrm{au}]$ & $63 \pm 18$ & $64 \pm 19$ \\
$r_{p, \text { outer }}[\mathrm{au}]$ & $45 \pm 21$ & $45 \pm 21$ \\
\hline
\end{tabular}

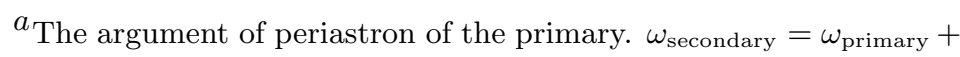
$\pi$.

$b_{\text {The ascending node is identified as the point where the secondary }}$ body crosses the sky plane receding from the observer.

${ }^{c}$ Posterior is non-Gaussian and not accurately represented by a summary statistic; see Figure 6

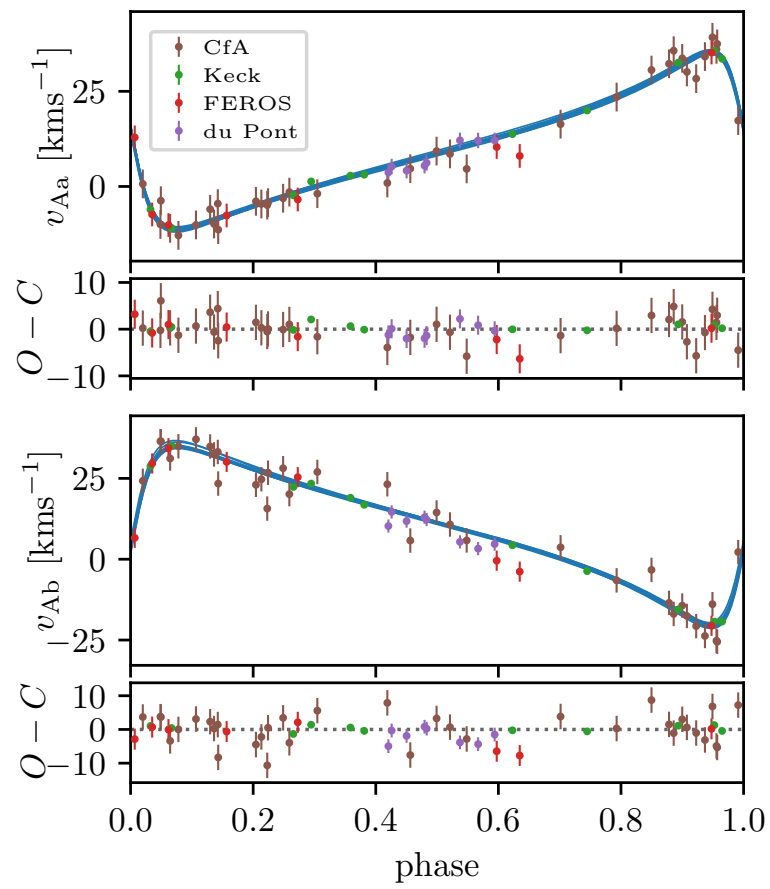

Figure 5. The phase-folded inner binary orbit and radial velocity residuals. In blue are ten realizations of the inner orbit with the velocity trend from B removed. The small scatter demonstrates that the inner orbital parameters are tightly constrained by the data.

ner plot in Figure 6 is valid for both scenarios. Representative outer orbits drawn from the posterior distribution are shown in Figure 7. Although the formal uncertainties and inferred jitter values of the Keck $v_{\mathrm{B}}$ measurements are large $\left(0.59 \mathrm{~km} \mathrm{~s}^{-1}\right.$ and $0.82 \mathrm{~km} \mathrm{~s}^{-1}$, respectively), the actual scatter of the four measured values is substantially smaller. The first two measurements (separated by 48 days in 2002/2003) differ by only $0.05 \mathrm{~km} \mathrm{~s}^{-1}$. The second two measurements (separated by one year in $2009 / 2010$ ) differ by only $0.15 \mathrm{~km} \mathrm{~s}^{-1}$. While circumstantial, this does raise the possibility that the uncertainties on the $v_{\mathrm{B}}$ measurements are overestimated (potentially driven by the scatter in $v_{\mathrm{Aa}}$ and $\left.v_{\mathrm{Ab}}\right)$ and that the increase in $v_{\mathrm{B}}$ over the Keck measurement baseline $\left(\approx 0.5 \mathrm{~km} \mathrm{~s}^{-1}\right.$ over 8 years $)$ may be significant. If true, a monotonically increasing $v_{\mathrm{B}}$ clearly favors a single posterior mode, highlighted in blue in Figures $6 \& 7$ (the mode corresponding to decreasing $v_{\mathrm{B}}$ is highlighted in orange). The MCMC samples and PyMC3 models corresponding to both scenarios are available online. ${ }^{9}$

We used these orbital posteriors and the inferred values of $i_{\text {disk }}$ and $\Omega_{\text {disk }}$ to calculate the mutual inclina-

\footnotetext{
${ }^{9}$ https://zenodo.org/record/4568830\#.YDvTb11Kida Czekala (2021) and https://github.com/iancze/TWA-3-orbit
} 


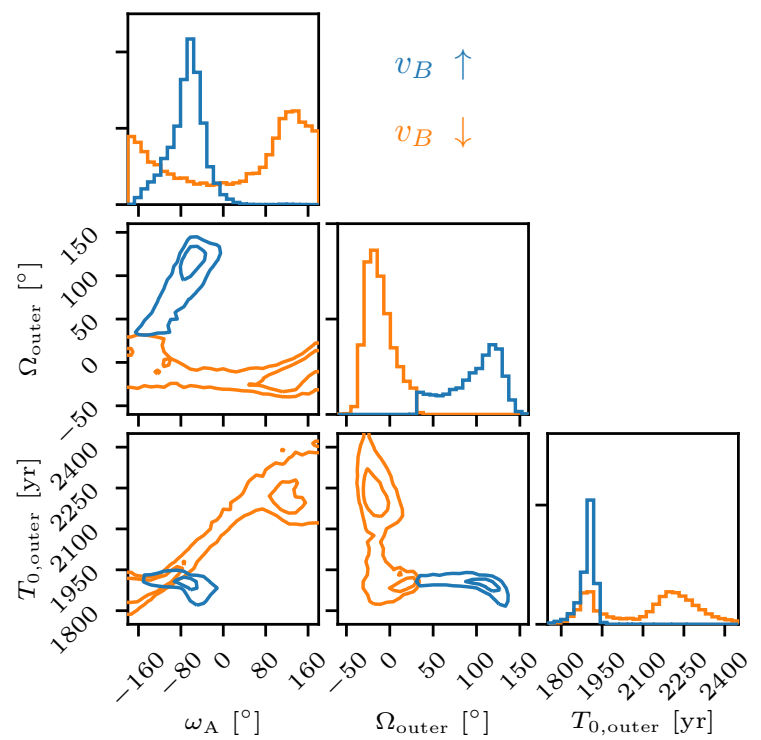

Figure 6. Corner plot of the sampled outer orbital parameters that have bimodal posterior distributions; contours are $1 \sigma$ and $2 \sigma$ within each mode. This figure is identical for both the "primary" and "alternate" solutions. Samples are color-coded based on whether they deliver increasing (blue) or decreasing (orange) $v_{\mathrm{B}}$ velocities over the Keck measurement baseline (see Figure 7). Note that the full posterior (the sum of the blue and orange contours) was sampled simultaneously, the samples have been bifurcated for plotting purposes only.

tions $\theta$ between the disk and the stellar orbits (e.g., Equation 1, Czekala et al. 2019) under different combinations of the degenerate orientations. ${ }^{10}$ As discussed in Czekala et al. (2019), the sensible imposition of spherically isotropic priors (i.e., the uniform priors on $\cos i_{\text {disk }}$ and $\left.\cos i_{\text {inner }}\right)$ results in effective mutual inclination priors of $p(\theta) \propto \sin (\theta)$. These isotropic priors have the consequence of strongly disfavoring coplanar architectures - simply because of the small phase space volume. To quantify the constraining power of the data only, we also report the posteriors re-weighted such that the effective prior is flat (i.e., similar to the marginal likelihood $p($ data $\mid \theta))$.

\section{DISCUSSION}

\subsection{Mutual inclinations}

10 Technically there are yet two more degenerate scenarios where $i_{\text {disk }}<90^{\circ}$ but $i_{\text {inner }}>90^{\circ}$, or vice-versa. Since the inferred inclinations of the inner binary and circumbinary disk are already so similar (modulo the degeneracy), we do not consider these scenarios.
As established in $\$ 3.3$, the inner binary $\mathrm{Aa}-\mathrm{Ab}$ orbit and circumbinary disk are very nearly coplanar with each other: across all scenarios listed in Table 4, $\theta_{\text {disk-inner }} \lesssim 6^{\circ}$ under a flat mutual inclination prior $\left(\theta_{\text {disk-inner }} \lesssim 13^{\circ}\right.$ under $\sin \theta_{\text {disk-inner }}$ prior $)$. Czekala et al. (2019) found that circumbinary planets, debris disks, and protoplanetary disks around short-period binaries $(P \lesssim 40$ days) all have low mutual inclinations. So, the low mutual inclination for the TWA $3 \mathrm{~A}$ is consistent with expectations given its 35-day orbital period.

That circumbinary disk mutual inclination trends with binary period is likely a byproduct of the formation mechanism for tight binary stars, which requires formation at larger distances and migration to present-day configurations (Bate et al. 2002). The close binary fractions of T Tauri stars and field stars are similar (Kounkel et al. 2019), implying that this migration occurs quickly, before the class II T Tauri phase. It is unlikely that tertiary interactions (e.g., Fabrycky \& Tremaine 2007) are responsible for the majority of tight binaries (Moe \& Kratter 2018); rather, migration via a circumbinary disk appears to be the dominant pathway (Tokovinin \& Moe 2020). TWA 3A represents both the lowest mass binary $\left(M_{\mathrm{A}}=0.53 \pm 0.01 M_{\odot}\right)$ hosting a circumbinary disk and the longest period binary ( $P_{\text {inner }}=34.879 \pm 0.001$ days $)$ before the population of mutual inclinations transitions from entirely coplanar systems to a broad dispersion of mutual inclinations (see Figure 14, Czekala et al. 2019). Though the range of binary periods over which this transition occurs is not yet well defined, recent observations have shed light on the dispersion of mutual inclinations at slightly longer binary periods of several months. The recent measurements of WW Cha by VLTI/GRAVITY have demonstrated that coplanar, truly low mutual inclination $\left(\theta<8^{\circ}\right)$ circumbinary disks can and do still

Table 4. Inferred mutual inclinations

\begin{tabular}{cccccc}
\hline \hline $\begin{array}{c}i_{\text {disk }} \\
\left.{ }^{\circ}\right]\end{array}$ & $v_{B}$ & $p(\theta)$ & $\begin{array}{c}\theta_{\text {disk-inner }} \\
\left.{ }^{\circ}\right]\end{array}$ & $\begin{array}{c}\theta_{\text {inner-outer }} \\
{\left[{ }^{\circ}\right]}\end{array}$ & $\begin{array}{c}\theta_{\text {disk-outer }} \\
{\left[^{\circ}\right]}\end{array}$ \\
\hline$<90$ & $\uparrow$ & $\sin (\theta)$ & $<8$ & $99_{-16}^{+17}$ & $99_{-17}^{+18}$ \\
$<90$ & $\uparrow$ & flat & $<3$ & $100_{-16}^{+17}$ & $100_{-18}^{+18}$ \\
$<90$ & $\downarrow$ & $\sin (\theta)$ & $<8$ & $140_{-10}^{+10}$ & $143_{-10}^{+7}$ \\
$<90$ & $\downarrow$ & flat & $<3$ & $142_{-11}^{+10}$ & $145_{-10}^{+7}$ \\
$>90$ & $\uparrow$ & $\sin (\theta)$ & $<13$ & $22_{-10}^{+14}$ & $20_{-12}^{+19}$ \\
$>90$ & $\uparrow$ & flat & $<6$ & $<20$ & $<15$ \\
$>90$ & $\downarrow$ & $\sin (\theta)$ & $<13$ & $76_{-17}^{+14}$ & $82_{-16}^{+13}$ \\
$>90$ & $\downarrow$ & flat & $<6$ & $75_{-17}^{+15}$ & $82_{-17}^{+14}$ \\
\hline
\end{tabular}

Note-One $\sigma$ asymmetric error bars are reported for all unimodal distributions. Sixty eight percent confidence upper limits are reported for one-sided distributions. 

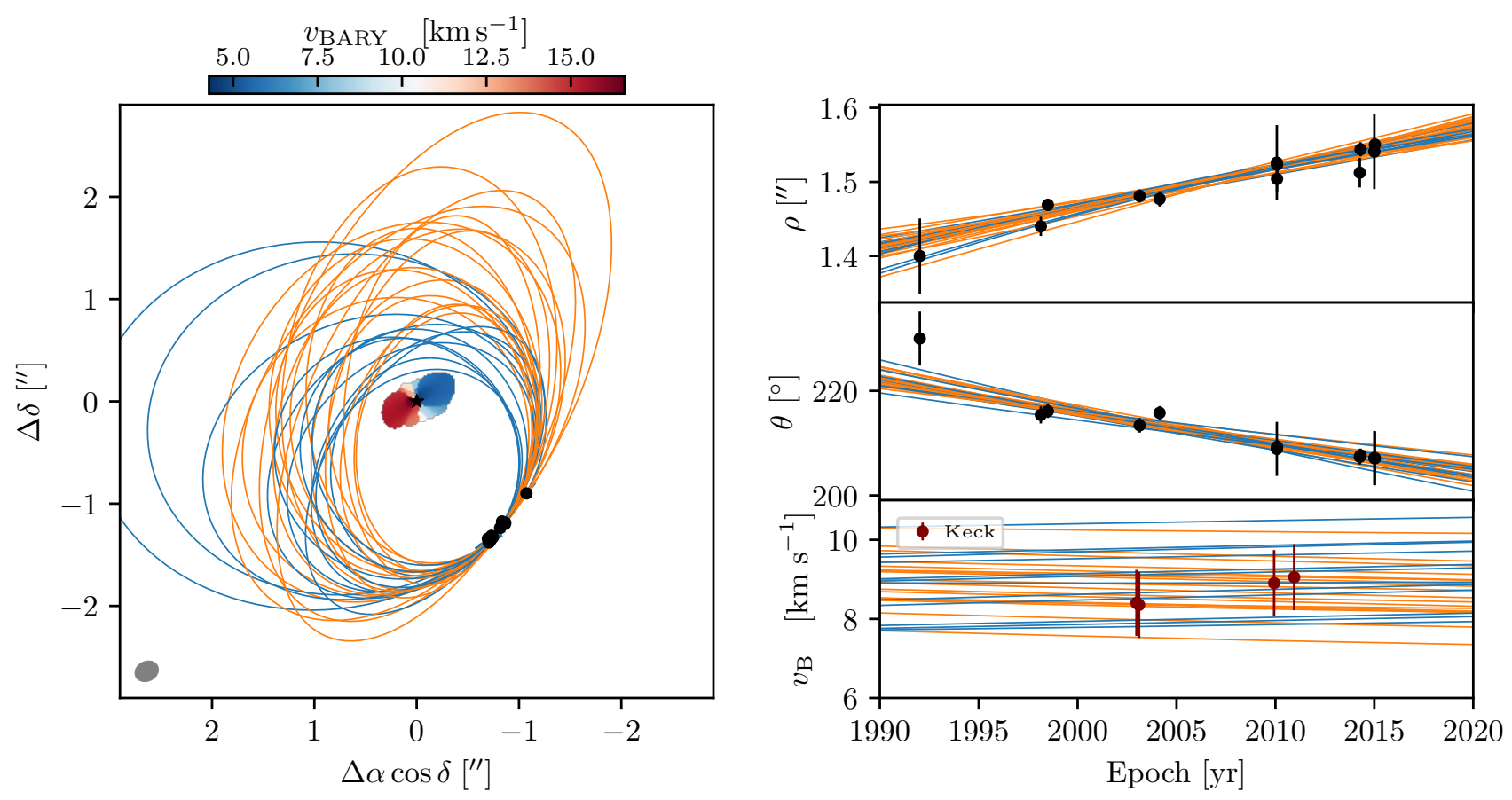

Figure 7. Thirty representative outer orbits drawn from the highlighted posterior modes in Figure 6 . left: the sky plane, centered on TWA 3A ( $\mathrm{Aa}$ and $\mathrm{Ab}$ are represented by the black star), with the the velocity field of the surrounding circumbinary disk. right: the astrometric data and Kellogg et al. (2017) Keck measurements of $v_{B}$. Orbits in blue correspond to solutions that deliver increasing $v_{B}$ over the Keck observation baseline; orange orbits are decreasing.

exist around longer period $(P=207$ days $)$, eccentric $(e=0.45)$ binaries (GRAVITY Collaboration et al. 2021). The measurement of WW Cha's orbital properties is important because it enhances the contrast of the stark transition from coplanar systems to a broad distribution of mutual inclinations. As a rule, all planets and disks orbiting binaries with $P \lesssim 40$ days are coplanar, yet at binary periods of $\sim 6-10$ months there already exists both a coplanar system (WW Cha) and a polar-oriented system (HD 98800B, $P=315$ days; Kennedy et al. 2019). Discovering new circumbinary disks with host binary periods near the "transition region" ( $P=30-300$ days) and measuring their mutual inclinations will help map out the regimes where migration may deliver a coplanar system.

Substantial degeneracies remain in the relationship between the inner and outer stellar orbits of TWA 3 . These degeneracies stem from two unknowns: 1) whether the circumbinary disk and inner binary are $i<90^{\circ}$ or $i>90^{\circ}$ and 2 ) which mode of the $\left(\omega_{\mathrm{A}}, \Omega_{\text {outer }}, T_{0, \text { outer }}\right)$ posterior is correct. The inferred mutual inclinations corresponding to the four permutations of these degeneracies are delineated in Table 4, under two different prior assumptions $(\sin (\theta)$ or flat). ${ }^{11}$ These separate into a coplanar configuration $\left(\theta_{\text {inner-outer }} \approx 0^{\circ}\right)$, two orthogonal configurations $\left(\theta_{\text {inner-outer }} \approx 90^{\circ}\right)$ and a retrograde configuration $\left(\theta_{\text {inner-outer }}>90^{\circ}\right)$. The field population of triples with projected outer separations $<50$ au exhibits a high degree of alignment between inner and outer orbital planes (average mutual inclination $<20^{\circ}$; Tokovinin 2017). Considering this backdrop, we suspect that the TWA 3 $\mathrm{Aa}-\mathrm{Ab}$ and $\mathrm{A}-\mathrm{B}$ orbital planes are nearly coplanar as well. We provide further hydrodynamical evidence for this scenario in the next subsection.

\subsection{Disk truncation}

The time-dependent gravitational potential of a binary star will influence the radial extent of a protoplanetary disk - clearing an interior cavity in a circumbinary configuration and truncating the outer edge in a circumstellar configuration. TWA 3 is noteworthy because both types of disk truncation are present in the same system.

Several analytical and numerical works have derived the radius (usually conveyed as a ratio relative to the binary semimajor axis, $r / a$ ) to which an interior, coplanar, and eccentric binary like $\mathrm{Aa}-\mathrm{Ab}\left(e_{\text {inner }}=0.63 \pm 0.01\right)$

\footnotetext{
11 Since the value of $\theta_{\text {disk-inner }}$ is low, values of $\theta_{\text {inner-outer }}$ and $\theta_{\text {disk-outer }}$ are similar in all cases. We will refer to $\theta_{\text {inner-outer }}$ in what follows but the points apply equally to $\theta_{\text {disk-outer }}$ as well.
} 
is predicted to clear the inner edge of circumbinary disk (Artymowicz \& Lubow 1994; Miranda \& Lai 2015; Miranda et al. 2017; Thun et al. 2017; Hirsh et al. 2020). Using an edge definition where the density falls to $50 \%$ of its peak value, Artymowicz \& Lubow (1994) found that $r / a \sim 2-3$, though they also noted that nonaxisymmetric waves at the rim of the disk make it difficult to define the edge location uniquely. Miranda \& Lai (2015) studied circumbinary disk truncation across a range of mutual inclinations, finding that the truncation radius was smaller for more misaligned systems. For coplanar systems, their results agreed with Artymowicz \& Lubow (1994). Using a suite of numerical simulations, Miranda et al. (2017) found that the truncation radius of coplanar circumbinary disks is $r / a \sim 1.7-2.6$ (using a $10 \%$ of peak density definition) though there is ambiguity in both the sharpness of the inner edge and peak location. Thun et al. (2017) used a 2D grid based setup to derive scalings for various inner edge thresholds. For an $e \approx 0.6$ binary, they found that the inner edge scales as $r / a \sim 3.5$ for a $10 \%$ of peak definition, but found that the $50 \%$ location scales like $r / a \sim 4-6$. More recently, Hirsh et al. (2020) used a smoothed-particle hydrodynamics (SPH) setup to derive $50 \%$ thresholds for an $e \approx 0.6$ binary, and found values more in line with the studies by Artymowicz \& Lubow (1994) and Miranda \& Lai $(2015): r_{\text {inner }} / a_{\text {inner }} \approx 3.0-3.5$. Hirsh et al. (2020) noted that Thun et al. (2017)'s discrepancy with previous results could be attributable to their choice of inner polar grid boundary. For the TWA 3A circumbinary disk, the best-fit interior cavity radius of $r_{\text {cav }} / a_{\text {inner }} \approx 3$ inferred from SED modeling $(\S 3.2)$ is squarely near the median of the aforementioned theoretical predictions, though solutions with $r_{\text {cav }} / a_{\text {inner }} \approx 4.5$ are still consistent with the SED data.

The inner edge of a circumbinary disk is also expected to be eccentric due to resonant interactions between the binary and the disk. For an $e_{\text {inner }}=0.63$ binary, Thun et al. (2017) found the inner edge would have $e_{\text {disk }} \approx 0.4$; Ragusa et al. (2020) (using SPH simulations of more extreme mass ratio binaries) also found similar results: $e_{\text {disk }} \approx 0.05-0.35$. Muñoz \& Lithwick (2020) used linear theory of perturbed, pressure-supported disks to solve for the eccentricity profile and showed that the eccentric modes are concentrated to within $r / a<2$ and drop off exponentially after $r / a \sim 10$ (corresponding to 1.7 au for TWA 3A). In simulations, the disk eccentricity is $e_{\text {disk }}<0.05$ after $r / a=10$ (Thun et al. 2017; Ragusa et al. 2020). Unfortunately, the scale of the inner rim of the circumbinary disk is below the resolution that can be meaningfully probed by the current ALMA observations. If the outer disk $(r \gtrsim 4 \mathrm{au})$ were eccentric, it would be readily observable as a strong flux asymmetry between the redshifted and blueshifted sides of the disk (Czekala et al. 2015). Such an effect is not seen in these observations.
For equal mass binaries, nearly circular $(e \lesssim 0.1)$, coplanar orbits are expected to truncate the outer edge of a circumstellar disk at radii $r_{\text {outer }} / a_{\text {outer }} \sim 0.32-0.38$ (Artymowicz \& Lubow 1994; Miranda \& Lai 2015). More eccentric binaries are more effective at truncating the disk: $r_{\text {outer }} / a_{\text {outer }} \sim 0.20-0.25$ for $e=0.2-$ 0.4. Coplanar configurations are also the most effective at truncating the disk: as the mutual inclination $\theta_{\text {inner-outer }}$ increases, $r_{\text {outer }}$ also increases (about $20 \%$ larger for $\theta_{\text {inner-outer }}=90^{\circ}$ and about $40 \%$ larger for $\theta_{\text {inner-outer }}=135^{\circ}$; see Figure 4 of Miranda \& Lai $2015)$. Using the $95 \%$ enclosed mass limit of $8.5 \pm 0.2 \mathrm{au}$ from the CO modeling and our orbital constraint of $a_{\text {outer }}=63 \pm 18$ au means that $r_{\text {outer }} / a_{\text {outer }} \approx 0.10-0.19$ for TWA 3.

To further investigate the role of external disk truncation in the TWA 3 system, we performed 3D SPH simulations of the interactions between the TWA 3A binary, circumbinary disk, and TWA 3B using the PHANTOM code (Price et al. 2018a). The SPH method is well suited for misaligned disk simulations given that there is no preferred geometry and angular momentum is conserved to the accuracy of the time-stepping scheme (see e.g. Price 2012). We used $10^{6}$ gas particles to model the circumbinary disc and set the initial inner and outer radii of the disk to $r_{\text {in }}=2$ au and $r_{\text {out }}=20 \mathrm{au}$, respectively. The surface density initially followed a power-law profile $\left(\Sigma \propto R^{-1}\right)$ and the temperature profile followed the power law profile $T=34 \mathrm{~K}(r / 10 \mathrm{au})^{-0.5}$, as in Price et al. (2018b). The disc total mass was set to $0.01 M_{\odot}$, which allowed us to neglect the disk self-gravity. Furthermore, we assumed that the disc is locally isothermal, where the sound speed follows a power-law $c_{\mathrm{S}} \propto r^{-1 / 4}$ with $H / r=0.05$ at $r=10$ au. Finally, we adopted a mean Shakura-Sunyaev disc viscosity $\alpha_{\mathrm{SS}} \approx 5 \times 10^{-3}$.

We included all three stars in the simulation (TWA 3Aa, Ab, and B, each represented by a sink particle), which interact with the gas via gravity and accretion (Bate et al. 1995). The accretion radii of $\mathrm{Aa}$ and $\mathrm{Ab}$ were set equal to $0.01 \mathrm{au}$, while the accretion radius of $\mathrm{B}$ was set equal to $10 \%$ of the Hill radius. These values ensure that the inner and outer regions of the disk are properly modeled while keeping computational costs reasonable (see e.g. Price et al. 2018b, Cuello et al. 2019 and Ménard et al. 2020).

The masses and orbits of stars $\mathrm{Aa}$ and $\mathrm{Ab}$ were initialized to the best-fit values listed in Table 3. The circumbinary disc around $\mathrm{Aa}$ and $\mathrm{Ab}$ was initially in the same plane as the $\mathrm{Aa}-\mathrm{Ab}$ binary orbit. The outer companion $\mathrm{B}$ was set on a circular $\left(e_{\text {outer }}=0\right)$ orbit with semi-major axis $a_{\text {outer }}$ and mutual inclination with respect to the binary orbital plane of $\theta_{\text {inner-outer }}$. In principle, the stellar orbits are allowed to change as mass is accreted, but since an insignificant amount of material was accreted throughout the simulation, the change in stellar orbits was negligible. We ran six simulations corresponding to the coplanar, orthogonal, and retrograde 


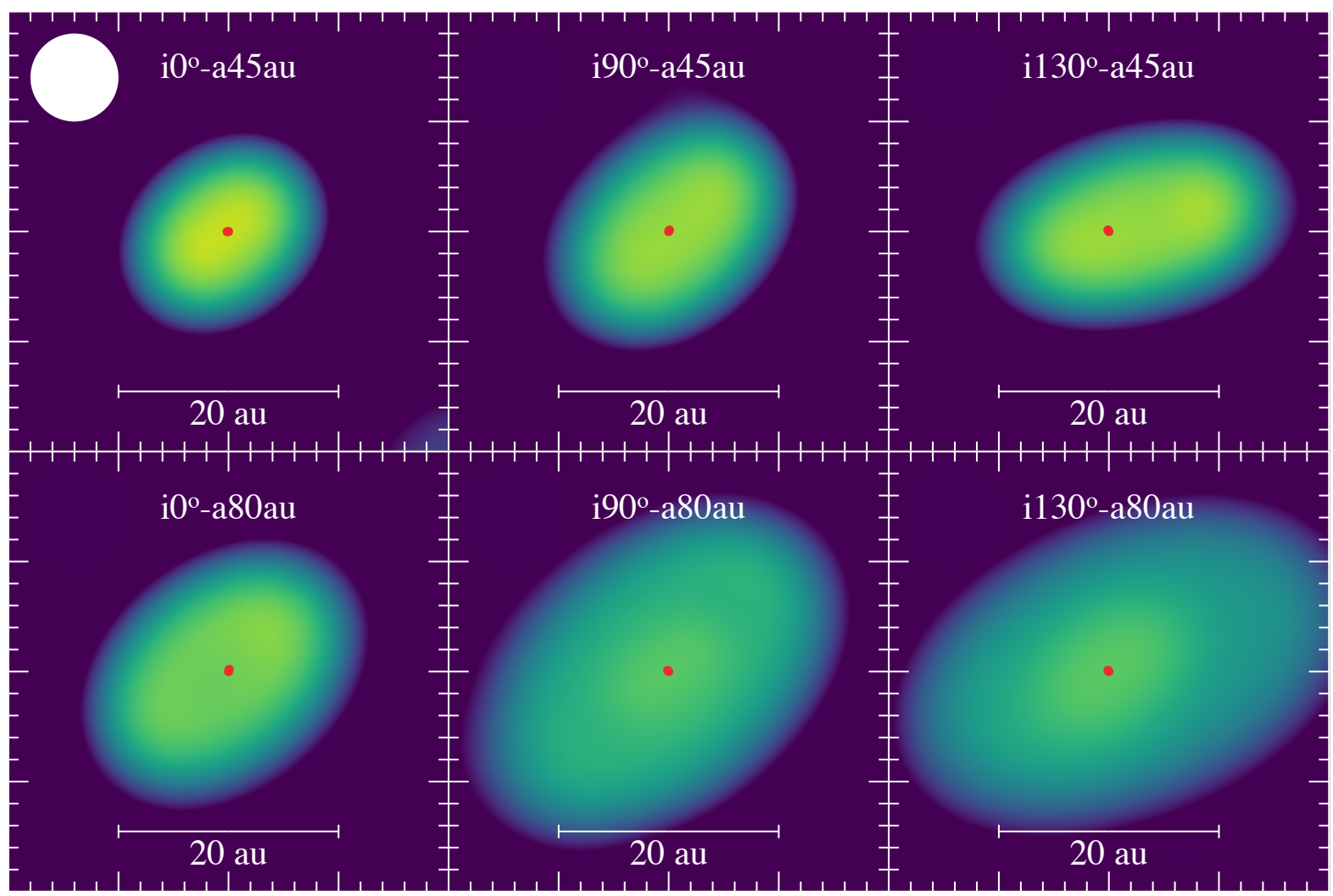

Figure 8. Gas surface density in SPH simulations of the TWA 3A binary, circumbinary disk, and exterior companion TWA 3B, over a

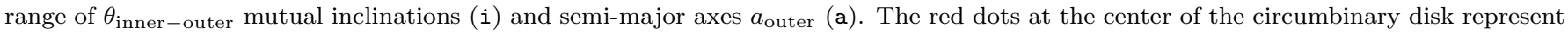
the positions of the stars $\mathrm{Aa}$ and $\mathrm{Ab}$ (on top of each other) after 5 orbits of the outer companion B (not shown in frame). The images are convolved with an 8 au Gaussian beam (white circle in the top left) for better comparison with the observations shown in Fig. 1. The orbit of $\mathrm{B}$ which most closely reproduces the outer radius of the circumbinary disk corresponds to the coplanar one with the smaller semi-major axis $\left(\theta_{\text {inner-outer }}=0^{\circ}, a_{\text {outer }}=45 \mathrm{au} ; \mathrm{i}^{\circ}-\mathrm{a} 45\right)$. Each representation is shown from the perspective of an Earth observer.

orbits of $\theta_{\text {inner-outer }}=\left[0^{\circ}, 90^{\circ}, 130^{\circ}\right]$ and two different values of $a_{\text {outer }}=[45 \mathrm{au}, 80 \mathrm{au}]$. We used $M_{\mathrm{B}}=0.4 M_{\odot}$ and $e_{\text {outer }}=0$ for all simulations. A visualization of the results (convolved with an 8 au beam) is provided in Figure 8, showing that the outer edge of the disk is sensitive to choices of $\theta_{\text {inner-outer }}$ and $a_{\text {outer }}$. The smallest disks were produced in the coplanar simulations $\left(\theta_{\text {inner-outer }}=0^{\circ}\right)$. The simulation parameters corresponding to the $i_{\text {disk }}>90^{\circ}$ and $v_{\mathrm{B}} \uparrow$ scenario $\left(\theta_{\text {inner-outer }}=0^{\circ}, a=45 \mathrm{au}, e=0\right.$ : i $0^{\circ}$-a $\left.45 \mathrm{au}\right)$ deliver an outer disk edge (10 au) that most closely matches the ALMA observations. This suggests that the true semimajor axis of the A-B binary lies closer to the lower range of its estimate $\left(a_{\text {outer }}=63 \pm 18 \mathrm{au}\right)$, the true eccentricity of the A-B binary is significantly non-zero $\left(e_{\text {outer }}=0.3 \pm 0.2\right)$, or both.

\subsection{Comparison to other systems}

The HD 98800 multiple system, coincidentally in the same TW Hydra association as TWA 3 (and thus, similarly aged at $\sim 10 \mathrm{Myr}$ ), is an apt analog system to TWA 3. Because the HD 98800B circumbinary disk is in a hierarchical multiple system, it also experiences both interior and exterior disk truncation forces (from the $\mathrm{Ba}-\mathrm{Bb}$ binary and from the wider $\mathrm{A}$ companion, respectively). Kennedy et al. (2019) spatially resolved the circumbinary disk with ALMA and convincingly demonstrated that it is in a circumpo$\operatorname{lar}\left(\theta_{\text {disk-inner }} \approx 90^{\circ}\right)$ configuration. Franchini et al. (2019) demonstrated that the relatively small cavity size $\left(r_{\text {cav }} / a_{\text {inner }} \sim 1.5-2.5\right)$ is a consequence of the reduced torques from an orthogonally-oriented binary (see also Miranda \& Lai 2015).

The exterior companion HD 98800A (which is itself a spectroscopic binary $\mathrm{Aa}-\mathrm{Ab}$, but here is effectively treated as a single star) orbits with $a_{\text {outer }}=54 \mathrm{au}$, 
$e=0.52$ and $\theta_{\text {outer }} \approx 65^{\circ} .{ }^{12}$ The HD $98800 \mathrm{~B}$ disk outer edge is $\lesssim 7 \mathrm{au}$, suggesting that the eccentric outer companion is much more effective at outer disk truncation $\left(r_{\text {outer }} / a_{\text {outer }} \sim 0.13\right)$, even though the disk and the outer companion are substantially misaligned. The disk may have survived as long as it has due to the combined effect of the inner binary stopping the accretion onto the central source (Kuruwita \& Federrath 2019) and the outer companion(s) stopping the viscous spreading of the disk (Ribas et al. 2018).

The HD 100453 multiple system is also a useful reference point for exterior disk truncation with a misaligned companion. The primary star HD 100453A $\left(1.7 M_{\odot}\right.$ A9Ve; Dominik et al. 2003) is surrounded by a disk whose $1.4 \mathrm{~mm}$ continuum emission extends to $\approx 40 \mathrm{au}$ and whose $\mathrm{CO}$ emission extends to $\approx 100 \mathrm{au}$ (Wagner et al. 2018; van der Plas et al. 2019). Scattered light observations of the disk revealed spiral arms (Wagner et al. 2015) and narrow-lane shadows (Benisty et al. 2017). HD $100453 \mathrm{~B}$ is an external $0.2 M_{\odot}$ companion located at $\approx 100$ au projected distance (Chen et al. 2006; Collins et al. 2009). Definitive conclusions about the influence of $\mathrm{B}$ on the disk are made difficult by the uncertainty in its orbit; however, recent analysis by Gonzalez et al. (2020) supports a scenario where the disk and binary plane are substantially misaligned $\left(60^{\circ}\right)$, since coplanar orbits consistent with the astrometric data would be otherwise inconsistent with the disk morphology, including the spirals and observed velocity field. The spiral features and narrow lane shadows seen in scattered light also suggest a complicated inner disk structure induced by an undetected, substellar companion interior to the disk (van der Plas et al. 2019; Rosotti et al. 2020; Nealon et al. 2020). The misalignments in this potentially triple system can be explained as being driven by the outer B, which drives the outer disk, substellar companion, and inner disk to precess and occasionally undergo KozaiLidov oscillations (Nealon et al. 2020).

The LTT 1445ABC triple system, which hosts a transiting exoplanet (Winters et al. 2019), also bears mentioning in the context of TWA 3. LTT 1445ABC consists of three mid to late $\mathrm{M}$ dwarfs in a hierarchical configuration: $\mathrm{B}-\mathrm{C}$ forms the inner binary and $\mathrm{A}$ is an outer tertiary and the most massive star in the system. The planet transits A; the entire stellar system is co-planar. The TWA 3 system is something of a pre-main sequence counterpoint to LTT $1445 \mathrm{ABC}$, in particular the configuration of its circumbinary disk contrasts with the fact that in LTT 1445A the planet transits the single star. Though if the TWA $3 \mathrm{Aa}-\mathrm{Ab}$ stars were considered together, TWA $3 \mathrm{~A}$ would also be the primary star in the system.

\footnotetext{
12 Kennedy et al. (2019) were unable to break the $i_{\text {disk }}<90^{\circ}$ or $i_{\text {disk }}>90^{\circ}$ degeneracy, but the computed value of $\theta_{\text {outer }}$ is similar for both cases.
}

\section{CONCLUSIONS}

Our main conclusions from this study of the TWA 3 system are as follows.

- We detected ${ }^{12} \mathrm{CO} J=2-1$ and ${ }^{13} \mathrm{CO} J=2-1$ emission from the TWA 3A circumbinary disk for the first time.

- We forward modeled the ${ }^{12} \mathrm{CO} J=2-1$ visibilities to derive an updated disk orientation $\left(\Omega_{\text {disk }}=\right.$ $116.5 \pm 0.4$ and $\left.i_{\text {disk }}=48.8 \pm 0.7\right)$ and infer the stellar mass enclosed by the disk $M_{\mathrm{A}}=M_{\mathrm{Aa}}+M_{\mathrm{Ab}}=$ $0.534 \pm 0.010$.

- We combined the disk dynamical constraints with extant radial velocity and astrometric measurements of TWA $3 \mathrm{Aa}, \mathrm{Ab}$, and $\mathrm{B}$ to infer individual stellar masses $0.29 \pm 0.01 M_{\odot}, 0.24 \pm 0.01 M_{\odot}$, and $0.40 \pm 0.28 M_{\odot}$, respectively.

- We drew constraints on the orbital architecture of the system, and inferred that the plane of the inner $\mathrm{Aa}-\mathrm{Ab}$ binary and its circumbinary disk are coplanar (misalignment $<6^{\circ}$ with $68 \%$ confidence). There are several degenerate solutions for the mutual inclination between the orbital planes of the inner $(\mathrm{Aa}-\mathrm{Ab})$ and outer $(\mathrm{A}-\mathrm{B})$ stellar orbits, however, SPH simulations lend support to the coplanar solution.
- We found the inner and outer radii of the circumbinary disk $(0.50-0.75$ au and $8.5 \pm 0.2 \mathrm{au}$, respectively) to be consistent with theoretical predictions of dynamical truncation from coplanar orbits. 


\section{ACKNOWLEDGMENTS}

IC would like to thank Rob De Rosa and Eric Nielsen for help with questions about orbital inference; Brian Mason for assistance with the Washington Double Star Catalog (Mason et al. 2001); Ryan Loomis for assistance with self-calibration; and Christophe Pinte for assistance with MCFOST. IC and AR would like to thank Elise Furlan for providing the calibrated IRS/Spitzer spectrum of TWA 3. IC was supported by NASA through the NASA Hubble Fellowship grant HST-HF2-51405.001-A awarded by the Space Telescope Science Institute, which is operated by the Association of Universities for Research in Astronomy, Inc., for NASA, under contract NAS526555. This project has received funding from the European Union's Horizon 2020 research and innovation programme under the Marie Skłodowska-Curie grant agreement No 210021. EC acknowledges NASA grants 80NSSC19K0506 and NNX15AD95G/NEXSS. The National Radio Astronomy Observatory is a facility of the National Science Foundation operated under cooperative agreement by Associated Universities, Inc. This paper makes use of the following ALMA data: ADS/JAO.ALMA\#2018.1.01545.S. ALMA is a partnership of ESO (representing its member states), NSF (USA) and NINS (Japan), together with NRC (Canada), MOST and ASIAA (Taiwan), and KASI (Republic of Korea), in cooperation with the Republic of Chile. The Joint ALMA Observatory is operated by ESO, AUI/NRAO and NAOJ. This research has made use of NASA's Astrophysics Data System Bibliographic Services.

Software: CASA (v4.4; McMullin et al. 2007), DiskJockey (Czekala et al. 2015; Czekala et al. 2019), RADMC-3D (Dullemond 2012), emcee (ForemanMackey et al. 2013), Astropy (Astropy Collaboration et al. 2013), PyMC3 (Salvatier et al. 2016), Theano (Theano Development Team 2016), MCFOST (Pinte et al. 2006), Phantom (Price et al. 2018a), SPlash (Price 2007), uvplot (Tazzari 2017) . 


\section{REFERENCES}

Aly, H., Dehnen, W., Nixon, C., \& King, A. 2015, MNRAS, 449, 65, doi: 10.1093/mnras/stv128

Andrews, S. M. 2020, ARA\&A, 58, 483, doi: 10.1146/annurev-astro-031220-010302

Andrews, S. M., Czekala, I., Wilner, D. J., et al. 2010, ApJ, 710, 462, doi: 10.1088/0004-637X/710/1/462

Andrews, S. M., Huang, J., Pérez, L. M., et al. 2018, ApJ, 869, L41, doi: 10.3847/2041-8213/aaf741

Anthonioz, F., Ménard, F., Pinte, C., et al. 2015, A\&A, 574, A41, doi: 10.1051/0004-6361/201424520

Artymowicz, P., \& Lubow, S. H. 1994, ApJ, 421, 651, doi: 10.1086/173679

Astropy Collaboration, Robitaille, T. P., Tollerud, E. J., et al. 2013, A\&A, 558, A33, doi: 10.1051/0004-6361/201322068

Bailer-Jones, C. A. L., Rybizki, J., Fouesneau, M., Mantelet, G., \& Andrae, R. 2018, AJ, 156, 58, doi: $10.3847 / 1538-3881 /$ aacb21

Bate, M. R. 2019, MNRAS, 484, 2341, doi: $10.1093 / \mathrm{mnras} / \mathrm{stz} 103$

Bate, M. R., Bonnell, I. A., \& Bromm, V. 2002, MNRAS, 336, 705, doi: 10.1046/j.1365-8711.2002.05775.x

Bate, M. R., Bonnell, I. A., \& Price, N. M. 1995, MNRAS, 277, 362, doi: 10.1093/mnras/277.2.362

Bell, C. P. M., Mamajek, E. E., \& Naylor, T. 2015, MNRAS, 454, 593, doi: 10.1093/mnras/stv1981

Benisty, M., Stolker, T., Pohl, A., et al. 2017, A\&A, 597, A42, doi: 10.1051/0004-6361/201629798

Brandeker, A., Jayawardhana, R., \& Najita, J. 2003, AJ, 126, 2009, doi: 10.1086/378057

Brogan, C. L., Hunter, T. R., \& Fomalont, E. B. 2018, arXiv e-prints, arXiv:1805.05266.

https://arxiv.org/abs/1805.05266

Chen, X. P., Henning, T., van Boekel, R., \& Grady, C. A. 2006, A\&A, 445, 331, doi: 10.1051/0004-6361:20054122

Collins, K. A., Grady, C. A., Hamaguchi, K., et al. 2009, ApJ, 697, 557, doi: 10.1088/0004-637X/697/1/557

Correia, S., Zinnecker, H., Ratzka, T., \& Sterzik, M. F. 2006, A\&A, 459, 909, doi: 10.1051/0004-6361:20065545

Cuello, N., \& Giuppone, C. A. 2019, A\&A, 628, A119, doi: 10.1051/0004-6361/201833976

Cuello, N., Dipierro, G., Mentiplay, D., et al. 2019, MNRAS, 483, 4114, doi: 10.1093/mnras/sty3325

Czekala, I. 2021, MCMC samples for TWA 3 orbital analysis, Zenodo, doi: 10.5281/zenodo.4568830

Czekala, I., Andrews, S. M., Jensen, E. L. N., et al. 2015, ApJ, 806, 154, doi: 10.1088/0004-637X/806/2/154

Czekala, I., Andrews, S. M., Torres, G., et al. 2016, ApJ, 818, 156, doi: 10.3847/0004-637X/818/2/156
Czekala, I., Chiang, E., Andrews, S. M., et al. 2019, ApJ, 883, 22, doi: 10.3847/1538-4357/ab287b

Czekala, I., Jensen, E., \& Huang, J. 2019, iancze/DiskJockey: Upgrades to Julia v1.0, doi: 10.5281/zenodo.3235028

Dominik, C., Dullemond, C. P., Waters, L. B. F. M., \& Walch, S. 2003, A\&A, 398, 607, doi: 10.1051/0004-6361:20021629

Dullemond, C. P. 2012, RADMC-3D: A multi-purpose radiative transfer tool, Astrophysics Source Code Library. http://ascl.net/1202.015

Fabrycky, D., \& Tremaine, S. 2007, ApJ, 669, 1298, doi: 10.1086/521702

Foreman-Mackey, D., Hogg, D. W., Lang, D., \& Goodman, J. 2013, PASP, 125, 306, doi: 10.1086/670067

Foreman-Mackey, D., Luger, R., Czekala, I., et al. 2020, exoplanet-dev/exoplanet v0.3.2, doi: 10.5281/zenodo.1998447

Foucart, F., \& Lai, D. 2013, ApJ, 764, 106, doi: 10.1088/0004-637X/764/1/106

Franchini, A., Lubow, S. H., \& Martin, R. G. 2019, ApJL, 880, L18, doi: 10.3847/2041-8213/ab2fd8

Gaia Collaboration, Brown, A. G. A., Vallenari, A., et al. 2018, A\&A, 616, A1, doi: 10.1051/0004-6361/201833051

Gonzalez, J.-F., van der Plas, G., Pinte, C., et al. 2020, MNRAS, 499, 3837, doi: 10.1093/mnras/staa2938

GRAVITY Collaboration, Eupen, F., Labadie, L., et al. 2021, arXiv e-prints, arXiv:2102.00122. https://arxiv.org/abs/2102.00122

Herczeg, G. J., \& Hillenbrand, L. A. 2014, ApJ, 786, 97, doi: 10.1088/0004-637X/786/2/97

Hirsh, K., Price, D. J., Gonzalez, J.-F., Ubeira-Gabellini, M. G., \& Ragusa, E. 2020, MNRAS, 498, 2936, doi: $10.1093 / \mathrm{mnras} / \mathrm{staa} 2536$

Hoffman, M. D., \& Gelman, A. 2011, arXiv e-prints, arXiv:1111.4246. https://arxiv.org/abs/1111.4246

Janson, M., Bergfors, C., Brandner, W., et al. 2014, ApJS, 214, 17, doi: 10.1088/0067-0049/214/2/17

Jayawardhana, R., Hartmann, L., Fazio, G., et al. 1999, ApJL, 521, L129, doi: 10.1086/312200

Kellogg, K., Prato, L., Torres, G., et al. 2017, ApJ, 844, 168, doi: 10.3847/1538-4357/aa7c60

Kennedy, G. M., Matrà, L., Facchini, S., et al. 2019, Nature Astronomy, 3, 230, doi: 10.1038/s41550-018-0667-x

Knapp, W., \& Nanson, J. 2018, Journal of Double Star Observations, 14, 503

Kounkel, M., Covey, K., Moe, M., et al. 2019, AJ, 157, 196, doi: $10.3847 / 1538-3881 / a b 13 b 1$ 
Kuruwita, R. L., \& Federrath, C. 2019, MNRAS, 486, 3647, doi: $10.1093 / \mathrm{mnras} /$ stz1053

Larson, R. B. 1969, MNRAS, 145, 271, doi: $10.1093 /$ mnras/145.3.271

Lindegren, L., Hernández, J., Bombrun, A., et al. 2018, A\&A, 616, A2, doi: 10.1051/0004-6361/201832727

Martin, R. G., \& Lubow, S. H. 2017, ApJL, 835, L28, doi: $10.3847 / 2041-8213 / 835 / 2 /$ L28

Mason, B. D., Hartkopf, W. I., Miles, K. N., et al. 2018, AJ, 155, 215, doi: 10.3847/1538-3881/aab9b8

Mason, B. D., Wycoff, G. L., Hartkopf, W. I., Douglass, G. G., \& Worley, C. E. 2001, AJ, 122, 3466, doi: $10.1086 / 323920$

McJunkin, M., France, K., Schneider, P. C., et al. 2014, ApJ, 780, 150, doi: 10.1088/0004-637X/780/2/150

McMullin, J. P., Waters, B., Schiebel, D., Young, W., \& Golap, K. 2007, in Astronomical Society of the Pacific Conference Series, Vol. 376, Astronomical Data Analysis Software and Systems XVI, ed. R. A. Shaw, F. Hill, \& D. J. Bell, 127

Ménard, F., Cuello, N., Ginski, C., et al. 2020, A\&A, 639, L1, doi: 10.1051/0004-6361/202038356

Miranda, R., \& Lai, D. 2015, MNRAS, 452, 2396, doi: 10.1093/mnras/stv1450

Miranda, R., Muñoz, D. J., \& Lai, D. 2017, MNRAS, 466, 1170, doi: 10.1093/mnras/stw3189

Moe, M., \& Kratter, K. M. 2018, ApJ, 854, 44, doi: 10.3847/1538-4357/aaa6d2

Muñoz, D. J., \& Lithwick, Y. 2020, ApJ, 905, 106, doi: 10.3847/1538-4357/abc74c

Nealon, R., Cuello, N., Gonzalez, J.-F., et al. 2020, MNRAS, 499, 3857, doi: 10.1093/mnras/staa2721

Offner, S. S. R., Dunham, M. M., Lee, K. I., Arce, H. G., \& Fielding, D. B. 2016, ApJL, 827, L11, doi: $10.3847 / 2041-8205 / 827 / 1 / \mathrm{L} 11$

Offner, S. S. R., Kratter, K. M., Matzner, C. D., Krumholz, M. R., \& Klein, R. I. 2010, ApJ, 725, 1485, doi: 10.1088/0004-637X/725/2/1485

Pinte, C., Dent, W. R. F., Ménard, F., et al. 2016, ApJ, 816, 25, doi: 10.3847/0004-637X/816/1/25

Pinte, C., Ménard, F., Duchêne, G., \& Bastien, P. 2006, A\&A, 459, 797, doi: 10.1051/0004-6361:20053275

Price, D. J. 2007, PASA, 24, 159, doi: 10.1071/AS07022

—. 2012, Journal of Computational Physics, 231, 759, doi: 10.1016/j.jcp.2010.12.011

Price, D. J., Wurster, J., Tricco, T. S., et al. 2018a, PASA, 35, e031, doi: 10.1017/pasa.2018.25

Price, D. J., Cuello, N., Pinte, C., et al. 2018b, MNRAS, 477, 1270, doi: 10.1093/mnras/sty647
Ragusa, E., Alexander, R., Calcino, J., Hirsh, K., \& Price, D. J. 2020, MNRAS, 499, 3362,

doi: $10.1093 / \mathrm{mnras} /$ staa2954

Reipurth, B., \& Zinnecker, H. 1993, A\&A, 278, 81

Remijan, A., Biggs, A., Cortes, P., et al. 2019, ALMA Cycle 7 Technical Handbook, doi: 10.5281/zenodo.4511522

Ribas, Á., Macías, E., Espaillat, C. C., \& Duchêne, G. 2018, ApJ, 865, 77, doi: 10.3847/1538-4357/aad81b

Rosotti, G. P., Benisty, M., Juhász, A., et al. 2020, MNRAS, 491, 1335, doi: 10.1093/mnras/stz3090

Salvatier, J., Wiecki, T. V., \& Fonnesbeck, C. 2016, PeerJ Computer Science, 2, e55

Sheehan, P. D., Wu, Y.-L., Eisner, J. A., \& Tobin, J. J. 2019, ApJ, 874, 136, doi: 10.3847/1538-4357/ab09f9

Tazzari, M. 2017, mtazzari/uvplot: v0.1.1, doi: $10.5281 /$ zenodo. 1003113

Theano Development Team. 2016, arXiv e-prints, abs/1605.02688. http://arxiv.org/abs/1605.02688

Thun, D., Kley, W., \& Picogna, G. 2017, A\&A, 604, A102, doi: 10.1051/0004-6361/201730666

Tofflemire, B. M., Mathieu, R. D., Herczeg, G. J., Akeson, R. L., \& Ciardi, D. R. 2017, ApJ, 842, L12, doi: $10.3847 / 2041-8213 / \mathrm{aa} 75 \mathrm{cb}$

Tofflemire, B. M., Mathieu, R. D., \& Johns-Krull, C. M. 2019, AJ, 158, 245, doi: 10.3847/1538-3881/ab4f7d

Tokovinin, A. 2017, ApJ, 844, 103, doi: $10.3847 / 1538-4357 /$ aa 7746

Tokovinin, A., Mason, B. D., Hartkopf, W. I., Mendez, R. A., \& Horch, E. P. 2015, AJ, 150, 50, doi: 10.1088/0004-6256/150/2/50

Tokovinin, A., \& Moe, M. 2020, MNRAS, 491, 5158, doi: $10.1093 / \mathrm{mnras} / \mathrm{stz} 3299$

Tripathi, A., Andrews, S. M., Birnstiel, T., \& Wilner, D. J. 2017, ApJ, 845, 44, doi: 10.3847/1538-4357/aa7c62

van der Plas, G., Ménard, F., Gonzalez, J. F., et al. 2019, A\&A, 624, A33, doi: 10.1051/0004-6361/201834134

Wagner, K., Apai, D., Kasper, M., \& Robberto, M. 2015, ApJL, 813, L2, doi: 10.1088/2041-8205/813/1/L2

Wagner, K., Dong, R., Sheehan, P., et al. 2018, ApJ, 854, 130, doi: 10.3847/1538-4357/aaa767

Weaver, E., Isella, A., \& Boehler, Y. 2018, ApJ, 853, 113, doi: 10.3847/1538-4357/aaa481

Webb, R. A., Zuckerman, B., Platais, I., et al. 1999, ApJL, 512, L63, doi: 10.1086/311856

Weintraub, D. A., Saumon, D., Kastner, J. H., \& Forveille, T. 2000, ApJ, 530, 867, doi: 10.1086/308402

Winters, J. G., Medina, A. A., Irwin, J. M., et al. 2019, AJ, 158, 152, doi: 10.3847/1538-3881/ab364d

Zanazzi, J. J., \& Lai, D. 2018, MNRAS, 473, 603, doi: $10.1093 /$ mnras/stx2375 
Zhu, Z., Zhang, S., Jiang, Y.-F., et al. 2019, ApJL, 877, L18, doi: 10.3847/2041-8213/ab1f8c 


\section{APPENDIX}

A. ${ }^{13} \mathrm{CO} J=2-1$ CHANNEL MAPS

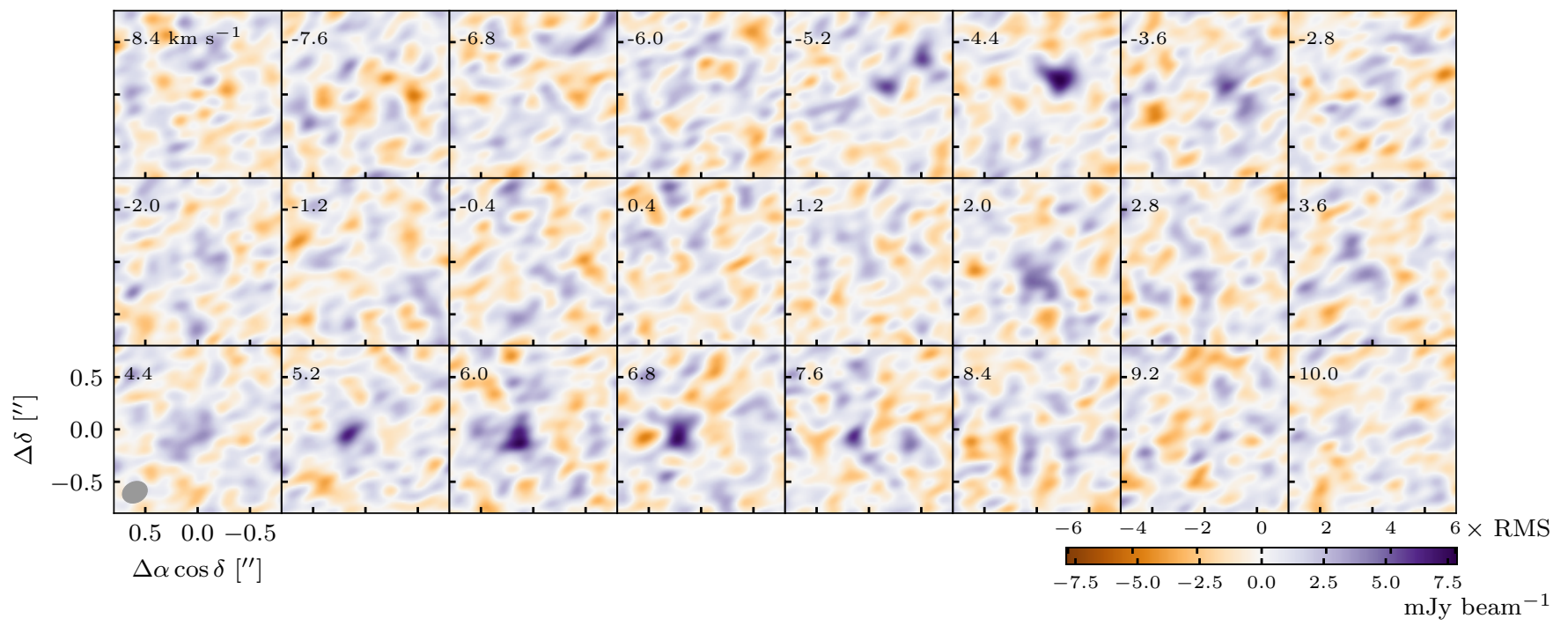

Figure 9. Continuum-subtracted ${ }^{13} \mathrm{CO} J=2-1$ data channel maps. Velocity scale is labeled in the LSRK frame. 